ARTICLE

\title{
Graphene overcoats for ultra-high storage density magnetic media
}

\author{
N. Dwivedi,2,10, A. K. Ott $3,4,10$, K. Sasikumar ${ }^{5}$, C. Dou ${ }^{3}$, R. J. Yeo ${ }^{2,6}$, B. Narayanan ${ }^{5}$, U. Sassi ${ }^{3}$, D. De Fazio (1) ${ }^{3}$, \\ G. Soavi ${ }^{3}$, T. Dutta ${ }^{2,7}$, O. Balci (10 ${ }^{3}$, S. Shinde ${ }^{3}$, J. Zhang ${ }^{3}$, A. K. Katiyar ${ }^{3}$, P. S. Keatley (1) ${ }^{8}$, A. K. Srivastava ${ }^{1}$, \\ S. K. R. S. Sankaranarayanan ${ }^{5,9}$, A. C. Ferrari (iD ${ }^{3 凶} \&$ C. S. Bhatia ${ }^{2}$
}

Hard disk drives (HDDs) are used as secondary storage in digital electronic devices owing to low cost and large data storage capacity. Due to the exponentially increasing amount of data, there is a need to increase areal storage densities beyond $1 \mathrm{~Tb} / \mathrm{in}^{2}$. This requires the thickness of carbon overcoats (COCs) to be $<2 \mathrm{~nm}$. However, friction, wear, corrosion, and thermal stability are critical concerns below $2 \mathrm{~nm}$, limiting current technology, and restricting COC integration with heat assisted magnetic recording technology (HAMR). Here we show that graphene-based overcoats can overcome all these limitations, and achieve two-fold reduction in friction and provide better corrosion and wear resistance than state-of-the-art COCs, while withstanding HAMR conditions. Thus, we expect that graphene overcoats may enable the development of 4-10 $\mathrm{Tb} / \mathrm{in}^{2}$ areal density HDDs when employing suitable recording technologies, such as HAMR and HAMR+bit patterned media

\footnotetext{
${ }^{1}$ CSIR-Advanced Materials and Processes Research Institute, Bhopal, India. ${ }^{2}$ Department of Electrical and Computer Engineering, National University of Singapore, Singapore, Singapore. ${ }^{3}$ Cambridge Graphene Centre, University of Cambridge, Cambridge, UK. ${ }^{4}$ Department of Engineering, University of Exeter, Exeter, UK. ${ }^{5}$ Center for Nanoscale Materials, Argonne National Laboratory, Argonne, IL, USA. ${ }^{6}$ Institute of Materials, Ecole Polytechnique Fédérale de Lausanne (EPFL), Lausanne, Switzerland. ${ }^{7}$ Empa-Swiss Federal Laboratories for Material Science and Technology, Dübendorf, Switzerland. ${ }^{8}$ Department of Physics and Astronomy, University of Exeter, Exeter, UK. ${ }^{9}$ Department of Mechanical and Industrial Engineering, University of Illinois, Chicago, IL, USA.

${ }^{10}$ These authors contributed equally: N. Dwivedi, A. K. Ott. ${ }^{凶}$ email: acf26@eng.cam.ac.uk
} 
$\mathrm{T}$ here has been an incessant increase in data generation over the past few decades ${ }^{1}$. The annual data creation rate was $\sim 16.1$ zettabytes/year $(\mathrm{ZB}, 1 \mathrm{ZB}=$ trillion gigabytes $(\mathrm{GB})$ ) in $2016^{1}$, and is expected to increase to $\sim 163 \mathrm{ZB} /$ year by $2025^{1}$. While various devices are used to store digital information, e.g., tape $^{2}$ and flash drives ${ }^{3,4}$, hard disk drives (HDDs) remain the primary choice as a secondary storage devices, due to their low cost $<0.1 \$ / G B$ at 2016 prices $^{3}$ and large storage capacity $>10 \mathrm{~TB}$ with 3.5 inch $\mathrm{HDDs}^{3}$. HDDs will rule storage technologies at least for the next 5-10 years ${ }^{5}$ in terms of capacity ${ }^{3,4}$, price ${ }^{3,4}$, production $^{3,4}$, and shipment ${ }^{3,4}$. Supplementary Note 1 provides the key background concepts on HDD technology and the main technological challenges.

Solid state drives ${ }^{3,4}$ are the main competing technology, posing a threat to future HDD viability ${ }^{4}$. Thus, novel technologies are needed to enable HDDs with high areal density $(\mathrm{AD})>1 \mathrm{~Tb} /$ in $^{23,6}$.

One option is to reduce the head-hard disk medium (HDM) spacing, since this decreases the signal-to-noise ratio ${ }^{6}$ and limits AD growth ${ }^{6}$. There are many contributors to the head-HDM spacing (Fig. 1a and Supplementary Note 1), whereby the HDM overcoat is the largest contributor.

Carbon-based overcoats (COCs) are widely used to protect HDM from mechanical damage ${ }^{7-12}$ and corrosion $8,13-15$, to ensure maintained functionality and durability ${ }^{13,14}$. By extrapolating Fig. 2 in ref. ${ }^{6}$, a COC thickness $<1 \mathrm{~nm}$ would be required for $\sim 10 \mathrm{~Tb} / \mathrm{in}^{2}$. However, current COCs lose most of their appealing properties, such as anti-friction ${ }^{11,12,15}$, wear resistance ${ }^{11,12}$, Young's Modulus ${ }^{7,8,13,16}$, and corrosion protection $7,8,13,16$, when their thickness is below 2-3 nm.

The ideal overcoat needs to provide: (1) corrosion protection, requiring complete coverage ${ }^{11,14}$ without pinholes ${ }^{13,14}$; (2) coefficient of friction (COF), i.e. the ratio of frictional to normal force, below $0.3-0.5$, lower than in $2.7 \mathrm{~nm}$ commercial COCs $s^{11,15}$ and wear resistance $^{11,12,14}$, requiring better lubricating properties ${ }^{11,12}$, hardness and elasticity with Young's modulus of at least $400 \mathrm{GPa}^{7,14}$; (3) lubricant compatibility ${ }^{14}$; (4) surface smoothness ${ }^{16-18}$, i.e., root mean square roughness $\sim 0.2-0.3 \mathrm{~nm}^{14}$. Given the limitations to achieve most of these properties with conventional COCs below 2 $\mathrm{nm}^{7,8,13,19}$, either the search for novel overcoats or engineering of existing overcoats is required to enable future ultra-high AD HDDs.

Another critical bottleneck hindering $\mathrm{AD}$ growth is the superparamagnetic limit ${ }^{20,21}$, which contributes to the magnetic trilemma, linking the three problems: $\mathrm{AD}$ increase, thermal stability, writability ${ }^{6,20-22}$ (see Supplementary Note 1).

To overcome writability issues in materials with high magnetic anisotropy, heat assisted magnetic recording (HAMR) was suggested ${ }^{23-25}$. HAMR uses a laser to heat the magnetic medium for $\sim 1 \mathrm{~ns}^{23}$ in order to decrease its coercivity, bringing the material to its Curie temperature, $T_{\mathrm{C}}$, above which it is writable with a magnetic field $\sim 0.8 \mathrm{~T}$ by conventional heads ${ }^{26}$. After cooling to room temperature, the coercivity goes back to its original value, and data is retained ${ }^{27}$. While HAMR appears to be a solution to all issues, such as small grain size, high magnetic anisotropy, and writability, and paves the way to increasing $\mathrm{AD}$, it raises concerns on the thermal stability of COCs against laser irradiation $^{28-30}$. As $T_{\mathrm{C}}$ is $\sim 700-750 \mathrm{~K}$ for $\mathrm{FePt}^{22,28,29}$, reached by laser irradiation, the overcoats for HAMR require thermal stability at least up to $\sim 700-800 \mathrm{~K}$ to avoid degradation over time $\mathrm{e}^{23,28,29}$.

Efforts are ongoing to test the thermal stability of HDM overcoats, e.g., by using laser ${ }^{28,29}$ and thermal annealing ${ }^{30}$, and by changing the thermal treatment time $e^{28,30,31}$ and heating rate ${ }^{28-31}$, to design HAMR-compatible overcoats. Reference 28 reported oxidation, degradation, and removal of a $\sim 5 \mathrm{~nm}$ hydrogenated amorphous carbon, a-C:H,-based commercial COC on HAMR- compatible HDM in HAMR-like conditions, with $0.25 \mathrm{~s}$ total irradiation time. Reference ${ }^{29}$ also reported degradation of $4 \mathrm{~nm}$ a-C:H-based commercial COC on a FePt-based HDM in HAMRlike conditions, with total heating time $\sim 0.1 \mathrm{~ms}$, corresponding to a 5-year drive life or $157.68 \times 10^{6} \mathrm{~s}^{29}$ (see Supplementary Note 1).

Better thermal stability was reported in filtered cathodic vacuum arc (FCVA)-based COCs under laser irradiation in HAMR-like conditions ${ }^{29}$ and thermal annealing up to $\sim 940 \mathrm{~K}^{32}$, consistent with the good thermal stability, i.e., no change in $s p^{3}$ content up to $1100^{\circ} \mathrm{C}$, found in tetrahedral a-C (ta-C) films ${ }^{33}$. However, FCVA-based COCs are not yet used as HDM overcoats due to the presence of macro-particles 6,34 .

Graphene is an emerging material for lubrication $35-39$, as well as oxidation ${ }^{40}$ and corrosion protection ${ }^{41-43}$. Reference ${ }^{37}$ reported that single-layer graphene (1LG) reduced the steel COF from 0.9 to 0.3 with a coating lifetime up to 6500 cycles, and decreased the wear rate by two orders of magnitude. Multilayer graphene (MLG) (3-4 layers) showed excellent tribological performance with a $\mathrm{COF}<0.2$, a decrease of wear rate by three orders of magnitude, and sliding lifetime up to 47000 cycles on steel $^{37}$. Reference ${ }^{35}$ reported that $1 \mathrm{LG}$ exhibits superlubricity ${ }^{35}$ and reduced the COF by 2-3 times and wear rate by two orders of magnitude for Au-based electrical contacts ${ }^{38}$. 1LG decreases the oxidation and corrosion of various metals, such as $\mathrm{Ni}^{40,41,43}$, $\mathrm{Co}^{41}, \mathrm{Fe}^{41}, \mathrm{Pt}^{41}, \mathrm{Cu}^{40,42,43}, \mathrm{Ag}^{42}$. Reduced graphene oxide was also used as a barrier coating ${ }^{44}$. Reference ${ }^{45}$ reported that suspended 1LG has good thermal stability up to $2600 \mathrm{~K}$, with a thermal conductivity up $\sim 2000 \mathrm{~W} / \mathrm{mK}^{46}$. In ref. ${ }^{47}$, we showed that it is possible to achieve high mobilities $\sim 30,000 \mathrm{~cm}^{2} \mathrm{~V} / \mathrm{s}$ at room temperature in wet transferred, polycrystalline 1LG. Thus, scalable processes, such as wet transfer, can be used for integration and packaging ${ }^{47,48}$. All these characteristics make 1 LG promising as a protective overcoat for both existing and HAMRbased technologies.

Here we use 1-4 layers of chemical vapor deposition (CVD) grown graphene (1-4LG) transferred on Co-alloy (current technology) and FePt-based (HAMR technology) HDMs, and test friction, wear, corrosion, thermal stability, and lube compatibility. We demonstrate that $1 \mathrm{LG}$ shows better performances than current $2.7 \mathrm{~nm}$ COCs on Co-alloy-based HDM, and good tribological properties with a $\mathrm{COF}<0.2$ for $>10,000$ cycles. We achieve very low $\mathrm{COF} \sim 0.15$ for $1 \mathrm{LG}$, indicating high wear resistance and corrosion protection, with corrosion current densities $<5 \mathrm{nA} / \mathrm{cm}^{2}$ for 2-3LG. Thermal stability tests confirm that $1 \mathrm{LG}$ on FePt can withstand HAMR-like conditions, without degradation. Graphene's superior performance and its thinness can enable the development of ultra-high-density magnetic data storage technologies, based either on current technology, as well as on HAMR, or HAMR combined with bit patterned media (BPM), where the magnetic storage layer is patterned into an array of pillars, each representing a single $\mathrm{bit}^{49}$. The combination of $1 \mathrm{LG}+\mathrm{HAMR}+\mathrm{BPM}$ may increase AD $>10 \mathrm{~Tb} / \mathrm{in}^{2}$.

\section{Results}

Magnetic media substrates. We use CoCrPt:Oxide-based bare HDM (BM) from Hitachi Global Storage Technologies (now Western Digital). These comprise multiple layers: seed bottom layer, soft-magnetic under layers (SUL), antiferromagnetic layer (A-FM), intermediate layers, and CoCrPt:Oxide storage layer (Fig. 1a, b). We also use other HDM from Western Digital with $\sim 2.7 \mathrm{~nm}$ commercial COC (CMC, Fig. 1c), and $\sim 2.7 \mathrm{~nm}$ commercial COC $+\sim 1 \mathrm{~nm}$ commercial PFPE lube (CMCL, Fig. 1d) as reference.

The details and nomenclature of the COCs are in Table 1. The structural properties of the FCVA and sputter-deposited COCs 

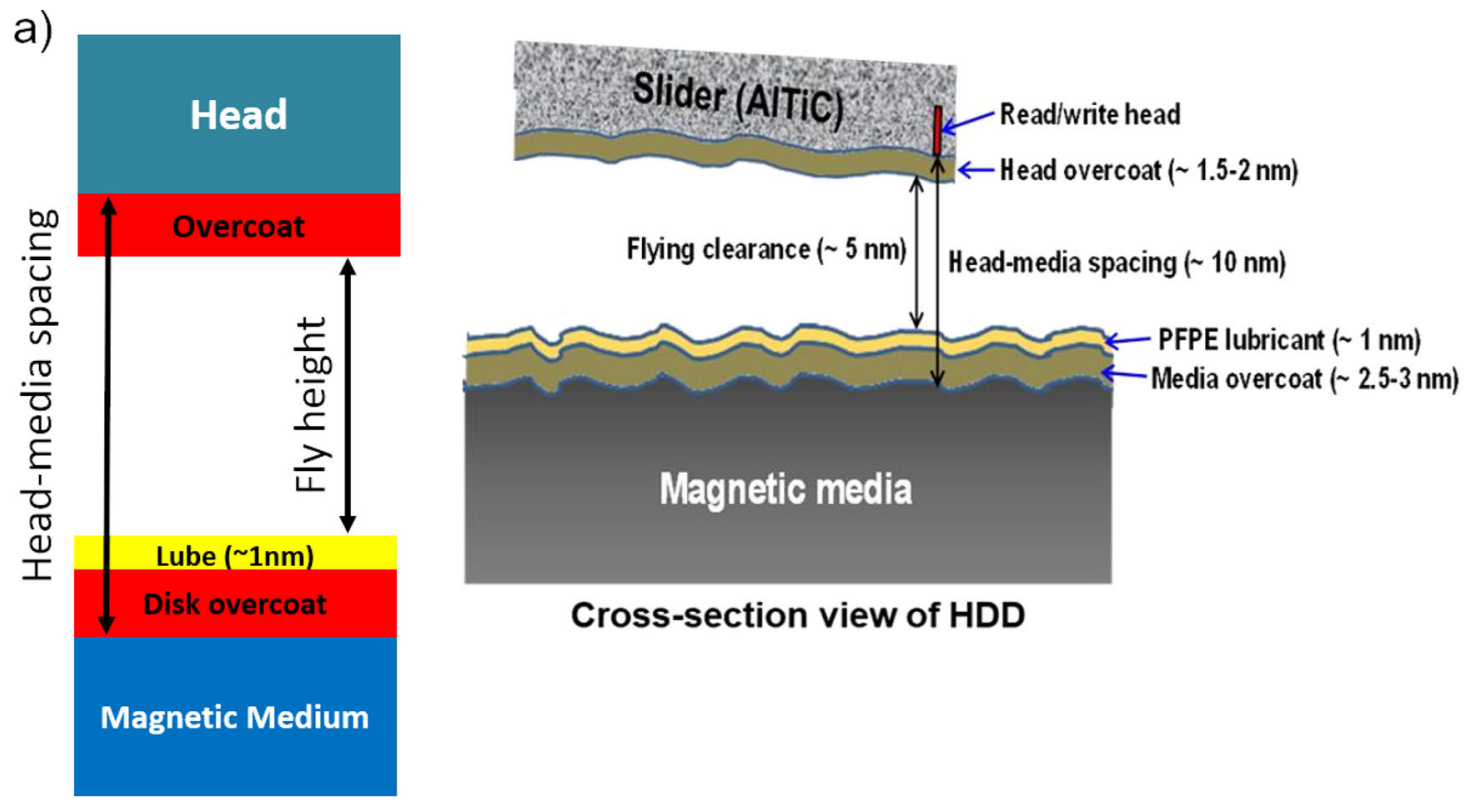

Cross-section view of HDD

\section{Co-alloy-based HDM}

b)

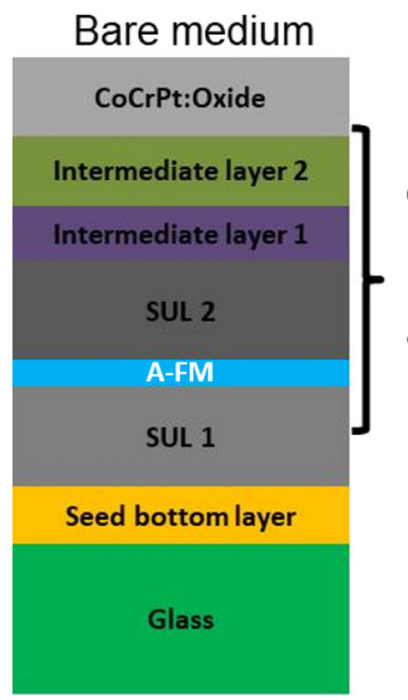

c)

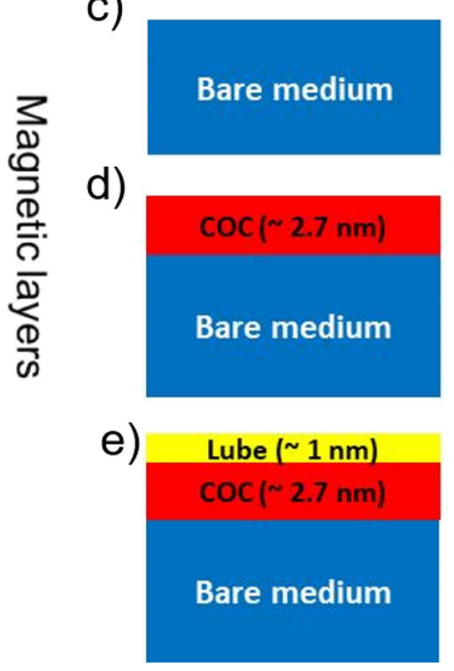

f)

\section{FePt-based HDM}

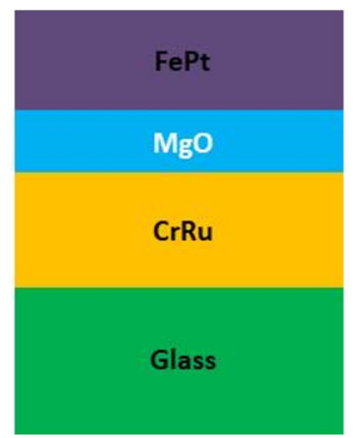

Fig. 1 Hard disk media structures. a Schematic cross-section of a hard disk drive with magnetic medium, disk overcoat, lubricant, fly height, head overcoat, head. The head-medium spacing and fly height/clearance are indicated by arrows. The slider containing the read/write head and the head overcoat are shown as well. For $1 \mathrm{~Tb} / \mathrm{in}^{2}$, the head-media spacing is $~ 8.9-6.5 \mathrm{~nm}^{6}$, based on the sum of overcoat thickness $(2.5-2 \mathrm{~nm})^{6}$, lubricant thickness $(1.2-1 \mathrm{~nm})^{6}$, touch down height $(2-1 \mathrm{~nm})^{6}$, fly clearance $(1.2-1 \mathrm{~nm})^{6}$ and head overcoat thickness $(2-1.5 \mathrm{~nm})^{6}$. b Full stack of bare CoCrPt:Oxide-based hard disk media comprising a glass substrate, seed bottom underlayer, and antiferromagnetic layer (A-FM) between two soft-magnetic under layers (SUL), followed by two intermediate layers and a co-based magnetic recording layer. c Bare medium (BM). d Magnetic medium with $2.7 \mathrm{~nm}$ commercial COC. e Magnetic medium with $\sim 2.7 \mathrm{~nm}$ commercial COC and $\sim \mathrm{nm}$ commercial lube. f Full stack of bare FePt-based hard disk medium (HDM) containing glass substrate, a layer of $\mathrm{CrRu}$ followed by layers of $\mathrm{MgO}$ and FePt.

can be found in refs. ${ }^{50,51}$. HAMR-compatible FePt-based HDMs are shown in Fig. 1f. See Supplementary Notes 2 and 3 for further details.

Graphene growth and transfer. Graphene is grown by chemical vapor deposition (CVD) and placed on the HMD by wet transfer 52,53 , as described in the "Methods" section. The quality and uniformity of the samples are assessed by Raman spectroscopy ${ }^{54}$. Unpolarized Raman spectra are recorded at 514.5, as well as at $785 \mathrm{~nm}$, close to the $850 \mathrm{~nm}$ used for HAMR writing tests ${ }^{25,55}$, with a Renishaw InVia spectrometer equipped with a Leica DM LM microscope and a $\times 100$ objective with a numerical aperture 0.85 . A $514.5 \mathrm{~nm}$ Raman spectrum of $1 \mathrm{LG}$ on $\mathrm{Cu}$ before the transfer is shown in Fig. 2a. The photoluminescence (PL) background due to the $\mathrm{Cu}$ foil is removed using baseline subtraction $^{56}$. The $\mathrm{D}$ to $\mathrm{G}$ intensity ratio $I(\mathrm{D}) / I(\mathrm{G})$ is $<0.1$, indicating a defect concentration $n_{d}<2.4 \times 10^{10} \mathrm{~cm}^{-2} 54,57-59$ (Fig. $2 \mathrm{~b}$ ). The $2 \mathrm{D}$ peak position can be fitted with a single Lorentzian with $\operatorname{Pos}(2 \mathrm{D}) \sim 2705 \mathrm{~cm}^{-1}$ and full-width-half-maximum, 


\section{Table 1 List of samples.}

BM CoCrPt-oxide-based BM without $\mathrm{COC}$ and lube

BML CoCrPt-oxide-based BM without COC, with PFPE lube $(1.2 \pm 0.2 \mathrm{~nm})$

CMC CoCrPt-oxide-based commercial HDM+COC $(\sim 2.7 \mathrm{~nm})$ without lube

CMCL CoCrPt-oxide-based commercial HDM+COC $(\sim 2.7 \mathrm{~nm})+$ PFPE lube $(\sim 1 \mathrm{~nm})$

$1 \mathrm{LG} \quad 1 \mathrm{LG}$ on CoCrPt-oxide-based commercial HDM

2LG 2LG on CoCrPt-oxide-based commercial HDM

3LG 3LG on CoCrPt-oxide-based commercial HDM

4LG 4LG on CoCrPt-oxide-based commercial HDM

$1 \mathrm{LGL} \quad$ PFPE lube $(1.2 \pm 0.2) \mathrm{nm}$-coated $1 \mathrm{LG}$ on CoCrPt-oxide-based commercial HDM

2LGL PFPE lube $(1.2 \pm 0.2) \mathrm{nm}$-coated $2 \mathrm{LG}$ on CoCrPt-oxide-based commercial HDM

3LGL PFPE lube $(1.2 \pm 0.2) \mathrm{nm}$-coated $3 \mathrm{LG}$ on CoCrPt-oxide-based commercial HDM

4LGL PFPE lube $(1.2 \pm 0.2) \mathrm{nm}$-coated $4 \mathrm{LG}$ on CoCrPt-oxide-based commercial HDM

3CF FCVA-deposited ta-C $(\sim 0.3 \mathrm{~nm})$ on CoCrPt-oxide-based commercial HDM

6CF FCVA-deposited ta-C $(\sim 0.6 \mathrm{~nm})$ on CoCrPt-oxide-based commercial HDM

12CF FCVA-deposited ta-C $(\sim 1.2 \mathrm{~nm})$ on CoCrPt-oxide-based commercial HDM

18CF FCVA-deposited ta-C $(\sim 1.8 \mathrm{~nm})$ on CoCrPt-oxide-based commercial HDM

8CS Pulsed DC sputtered $s p^{2}$ rich carbon $(\sim 0.8 \mathrm{~nm})$ on $\mathrm{CoCrPt}$-oxide commercial HDM

12CS Pulsed DC sputtered $s p^{2}$ rich carbon $(\sim 1.2 \mathrm{~nm})$ on CoCrPt-oxide commercial HDM

$\mathrm{FePt} \quad \mathrm{FePt} / \mathrm{MgO} / \mathrm{CrRu} / \mathrm{glass}$ samples grown by magnetron sputtering. $\mathrm{CrRu}$ and $\mathrm{MgO}$ seed layers are grown at $400^{\circ} \mathrm{C}$ and $1.5 \mathrm{mTorr}$; FePt is grown at $600^{\circ} \mathrm{C}$ and $3.5 \mathrm{mTorr}$ as discussed in ref. ${ }^{22}$.

Bare media, commercial media with an overcoat, graphene-coated media, FCVA, and sputtered carbon overcoats with and without lubricant (indicated by L), with details on sample structure and corresponding acronyms.
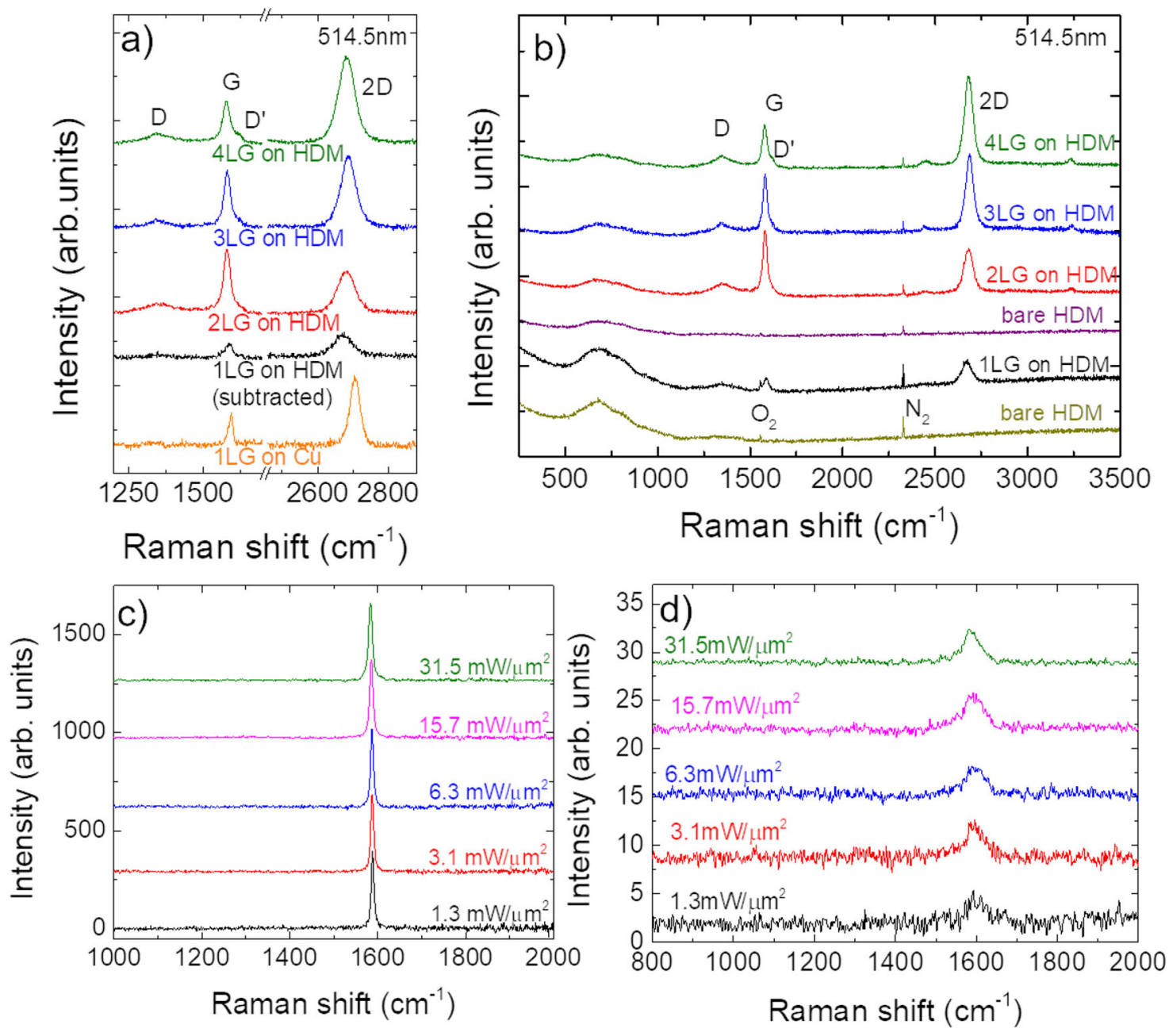

Fig. 2 Raman and irradiation measurements. a $514.5 \mathrm{~nm}$ Raman spectra of as-grown 1LG on Cu (before transfer) and 1-4LG transferred on CoCrPt:Oxide after CoCrPt:Oxide background subtraction. b $514.5 \mathrm{~nm}$ Raman spectra of CoCrPt:Oxide substrates and 1-4LG transferred onto CoCrPt:Oxide. c, d $785 \mathrm{~nm}$ Raman spectra of $1 \mathrm{LG}$ on (c) $\mathrm{Si}_{\mathrm{SiO}}$ and (d) FePt-based HAMR-HDM, for different laser power densities. 
$\operatorname{FWHM}(2 \mathrm{D}) \sim 33 \mathrm{~cm}^{-1}$, a signature of $1 \mathrm{LG}^{54,60}$. The $\mathrm{G}$ peak position and FWHM(G) are $\sim 1593 \mathrm{~cm}^{-1}$ and $16 \mathrm{~cm}^{-1}$. $I(2 \mathrm{D}) / I(\mathrm{G})$ and $A(2 \mathrm{D}) / A(\mathrm{G})$ are $\sim 2.3$ and 4.9 .

Representative Raman spectra of 1-4LG transferred on HDM are in Fig. 2a, with the HDM spectrum subtracted. The HDM spectrum and the spectra of 1L-4LG on HDM before background subtraction are in Fig. 2b. By subtracting the reference spectrum, using the $\mathrm{N}_{2}$ Raman peak from the air for normalization, from the spectrum taken on 1LG-coated HDM, we can reveal the 1LG contribution. After transfer, all peaks are downshifted and broader, with $\operatorname{Pos}(2 \mathrm{D})_{1 \mathrm{LG}} \sim 2672 \mathrm{~cm}^{-1}, \operatorname{Pos}(2 \mathrm{D})_{2 \mathrm{LG}} \sim 2681 \mathrm{~cm}^{-1}$, $\operatorname{Pos}(2 \mathrm{D})_{3 \mathrm{LG}} \sim 2687 \mathrm{~cm}^{-1}, \quad \operatorname{Pos}(2 \mathrm{D})_{4 \mathrm{LG}} \sim 2682 \mathrm{~cm}^{-1}, \quad$ FWHM (2D) ${ }_{1 \mathrm{LG}} \sim 61 \mathrm{~cm}^{-1}, \operatorname{FWHM}(2 \mathrm{D})_{2 \mathrm{LG}} \sim 57 \mathrm{~cm}^{-1}, \operatorname{FWHM}(2 \mathrm{D})_{3 \mathrm{LG}}$ $\sim 49 \mathrm{~cm}^{-1}, \operatorname{FWHM}(2 \mathrm{D})_{4 \mathrm{LG}} \sim 53 \mathrm{~cm}^{-1}$. Assuming $2686 \mathrm{~cm}^{-1}$ as unstrained $\operatorname{Pos}(2 \mathrm{D})^{61}$ and a rate of change in $\operatorname{Pos}(2 \mathrm{D})$ with strain $\delta \operatorname{Pos}(2 \mathrm{D}) / \delta \epsilon \sim-64 \mathrm{~cm}^{-1} / \%{ }^{61}$, this would give $\sim 0.22 \%$ uniaxial strain in $1 \mathrm{LG}$, consistent with the large $\operatorname{FWHM}(2 \mathrm{D})^{61,62}$. We assume the strain to be mostly uniaxial, because it is unlikely to have biaxial, i.e., perfectly isotropic strain, in all directions, unless this is induced on purpose. There is a small increase in $I(D) / I(G)$ to $\sim 0.2$ for $2-4 \mathrm{LG}$ on HDM.

Current commercial HDD technology uses a $\sim 2.5-3 \mathrm{~nm}$ COC deposited by plasma-based processes ${ }^{12,63}$. The thickness and roughness of 1-4LG are measured by Atomic Force Microscopy (AFM), as discussed in Supplementary Note 5. This shows that the thickness of 4LG on HDM is $\sim 2.1 \mathrm{~nm}$ (Supplementary Fig. 3), well below that of the current commercial COC. Graphene is transferred without such an energetic process as that used for commercial COC deposition. Thus, it does not adversely affect the magnetic performance. Even considering that COCs slightly affect the HDM magnetic properties, the influence of graphene will be much less than commercial COCs deposited by energetic processes, where energetic atoms/ions (neutrals also) hit and participate in deposition onto the HDM. Reference ${ }^{64}$ reported that 1LG grown by CVD on Co (and Ni) does not affect the magnetic properties of these materials. In order to confirm this in our case, we perform polar magneto-optical Kerr effect measurements on BM, BM covered with $\sim 2-3 \mathrm{~nm}$ COC, and BM covered with 1-4LG. 1-4LG coating results in a small change in the hysteresis loop. The coercivity changes from $510 \mathrm{mT}(\mathrm{BM})$ to $\sim 545 \mathrm{mT}$ (1-4LG and COC). The remanent magnetization is unchanged at $\sim 97-98 \%$ of the saturation magnetization when compared to BM. The coercivity and the remanent magnetization of 1-4LG-coated $\mathrm{BM}$ are similar to $\mathrm{BM}+\mathrm{COC}$, indicating that graphene coating does not have any detrimental effect on the media, see Supplementary Note 6 for details.

Laser irradiation stability. HAMR is not possible without laser heating. References $22,25,65$ reported that FePt does not degrade upon laser heating, with lubricant and COC being the main concerns when it comes to laser irradiation. Consistent with refs. ${ }^{25,55}$, here we use an IR laser to examine the $1-4 \mathrm{LG}$ irradiation stability. To test whether 1LG can withstand HAMR conditions we consider $1 \mathrm{LG}$ transferred on $\mathrm{L}_{0}$-FePt-based $\mathrm{HDM}^{25,27,55}$ and on $\mathrm{Si} / \mathrm{SiO}_{2}$. Reference ${ }^{55}$ achieved $T_{c}$ at $\sim 1.3 \mathrm{~mW} / \mu \mathrm{m}^{2}$ by optimizing aperture optics. Reference 25 suggested using a laser power density $\sim 10^{7} \mathrm{~W} / \mathrm{cm}^{2}$ and $\sim 0.35 \times$ $10^{7} \mathrm{~W} / \mathrm{cm}^{2}$ for FePt-based HDM. We perform Raman measurements at $785 \mathrm{~nm}$, the available Raman wavelength closest to that used in HAMR $\left(\sim 830 \mathrm{~nm}^{25,55}\right)$, with a spot size $\sim 1.24 \mu \mathrm{m}$, as determined by the razor blade technique, see Supplementary Note 7 for details. We vary the power density from $\sim 1.3 \mathrm{~mW} / \mu \mathrm{m}^{2}$ $\left(0.013 \times 10^{7} \mathrm{~W} / \mathrm{cm}^{2}\right)$ to $\sim 31.5 \mathrm{~mW} / \mu \mathrm{m}^{2}\left(0.315 \times 10^{7} \mathrm{~W} / \mathrm{cm}^{2}\right)$ in order to examine the laser irradiation-driven evolution of the SLG Raman spectrum under HAMR power densities.
We first consider $\mathrm{Si} / \mathrm{SiO}_{2} / 1 \mathrm{LG}$. We record spectra at different power densities for $\sim 4 \mathrm{~min}$ to achieve a good signal-to-noise ratio. Figure $2 \mathrm{c}$ shows that $1 \mathrm{LG}$ on $\mathrm{Si} / \mathrm{SiO}_{2}$ has no $\mathrm{D}$ peak even for the highest power density. Pos $(\mathrm{G})$ downshifts from $\sim 1588$ to $1583 \mathrm{~cm}^{-1}$, indicating an increase in $T \sim 312.5 \mathrm{~K}$ with respect to room temperature, by taking $\sim-0.016 \mathrm{~cm}^{-1} / \mathrm{K}$ as a shift of $\operatorname{Pos}(\mathrm{G})$ with $T^{66}$. Figure $2 \mathrm{~d}$ plots the data for $1 \mathrm{LG}$ on FePt HDM, with $I(\mathrm{G})$ normalized to that at $1.3 \mathrm{~mW} / \mu \mathrm{m}^{2}$. In this case, the FePt substrate is rotated at $4100 \mathrm{rpm}$ on a circular track with a diameter $\sim 4 \mathrm{~mm}$ to simulate rotating HDD conditions, with a $20 \mathrm{~min}$ acquisition time, much larger than the total laser irradiation time expected for a 5-years life of HAMR-based HDDs ${ }^{29}$. No D peak is seen for all power densities, thus confirming the stability of 1LG. The Raman data show that no visible changes occur during the irradiation test measurements.

Friction and wear. The HDM comprises magnetic/metallic layers, including Co-alloys-based magnetic storage layers ${ }^{14,20}$, with a high COF $\sim 0.6-0.8^{11,15}$ and wear ${ }^{11,15}$. Therefore, they can experience mechanical damage whenever intermittent contact occurs with the head ${ }^{11,15}$, and are susceptible to corrosion ${ }^{8,11,14}$ and oxidation $^{11,15}$, leading to HDD degradation or damage. The current $\sim 2.7 \mathrm{~nm}$ commercial COCs have a high COF $\sim 0.3-0.5^{11,15}$ and wear in a ball-on-disk tribological environment ${ }^{11,15}$, which can result in damage, hence durability concerns, see Supplementary Note 1 for more details.

Reference ${ }^{11}$ used FCVA to deposit $\sim 1.5-2 \mathrm{~nm}$ carbon films for protection of Co-alloy-based HDM and reported low COF $\sim 0.25$, wear, and corrosion. Here, we use 1-4LG grown by CVD for Coalloy-based and HAMR HDDs.

Since in HDDs the HDM spins during operation, COF and wear need to be examined in a setup mimicking HDD operation. AFM and other tip-based tools are used to measure COF and wear $^{39}$. In contrast to setups with rotating geometry, tip-based tools measure the COF based on the movement of the tip in the lateral direction ${ }^{39}$. Ball-on-disk measurements (rotation-based geometry) have a similar assembly as HDDs, with samples rotating while the counterface is in contact with the surface ${ }^{11,35}$, thus measuring COF. We perform ball-on-disk tests using a nano-tribometer (CSM Instruments) in a cleanroom, to have a controlled environment with $T=23 \pm 1^{\circ} \mathrm{C}$ and a relative humidity $\sim 55 \pm 5 \%$. A sapphire ball $\left(\mathrm{Al}_{2} \mathrm{O}_{3}\right)$ of diameter $\sim 2.0 \pm$ $0.1 \mathrm{~mm}$ and surface roughness $\sim 5.0 \pm 0.1 \mathrm{~nm}$ is used as the counterface because the hard disk head is made of an $\mathrm{Al}_{2} \mathrm{O}_{3}$ based composite ${ }^{6}$. During the test, a normal load $\sim 20 \mathrm{mN}$ and a rotational speed $\sim 100 \mathrm{rpm}$ are used, corresponding to a linear speed $\sim 1.05 \mathrm{~cm} / \mathrm{s}$ for 10,000 cycles.

Since in HDDs the contact occurs occasionally ${ }^{6}$, the 10,000 cycles in our setup are much higher than the HDD operational lifetime. After each test, the wear track and ball images are captured using an optical microscope. To check repeatability, tests are performed 2-7 times. When two surfaces are in contact, and at least one of the surfaces starts to slide with respect to the other, friction and wear occur ${ }^{67}$. As a result, a wear track is formed.

Figure 3 plots representative friction curves for BM, CMC, CMCL, and BML (Fig. 3a) coated with 1-4LG (Fig. 3b), and BM coated by FCVA (Fig. 3c). The average COFs are in Fig. 4, including HDM coated by DC-sputtering. BM has the highest $\mathrm{COF} \sim 0.8$, with substantial wear, as confirmed by optical images of balls and wear tracks in Figs. 5 and 6. The COF of $\sim 2.7 \mathrm{~nm} \mathrm{CMC}$ reaches $\sim 0.4$ at 10,000 cycles, but with strong fluctuations between $\sim 0.2$ and 0.6 at 1500 up to $\sim 3500$ cycles (Fig. 3a), and negligibly improves wear with respect to BM (Fig. 3a). The transfer of 1-4LG reduces $\mathrm{COF}<0.2$ for all samples, and gives non-fluctuating, smooth friction curves. The COF for 1-4LG-coated samples 


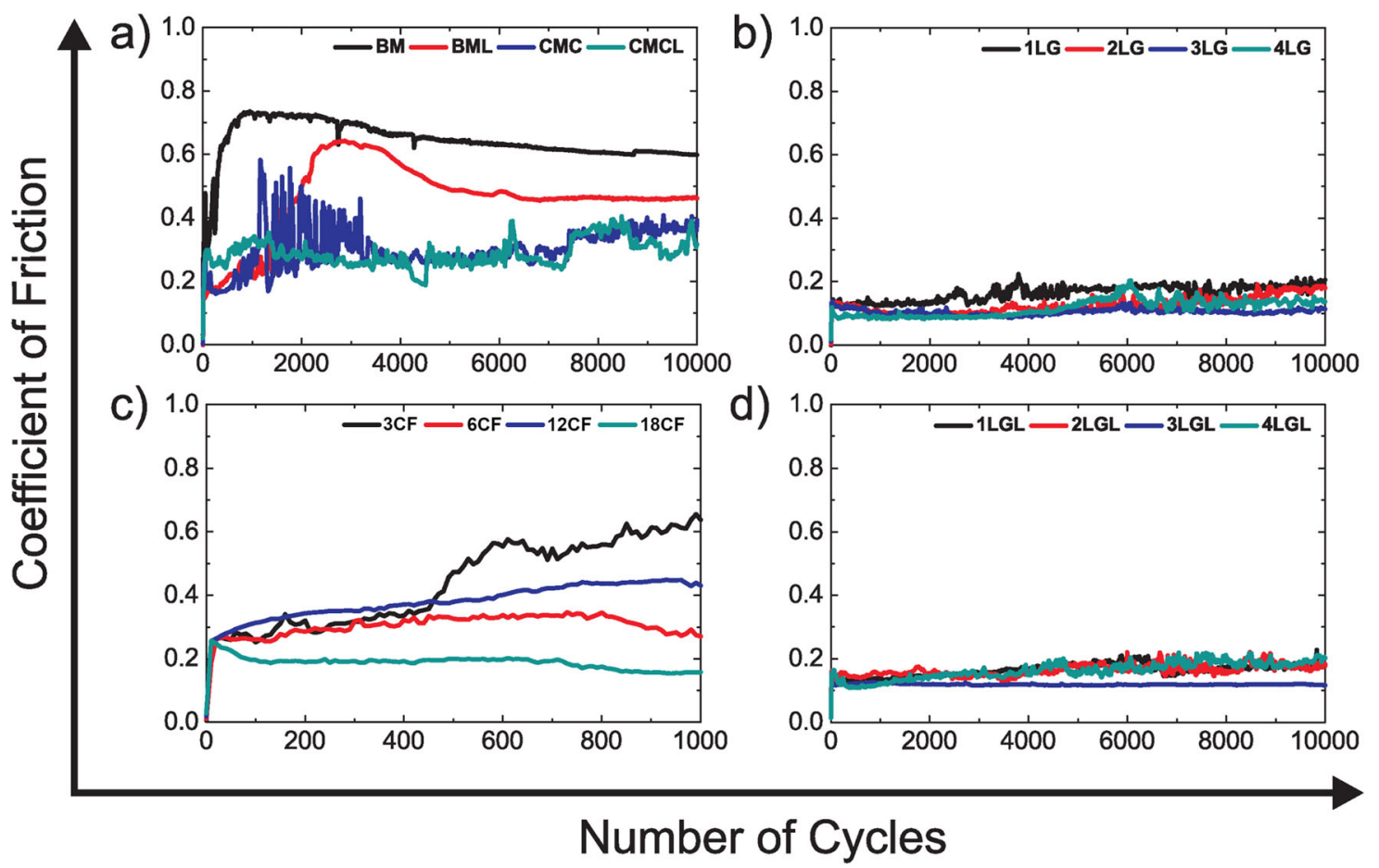

Fig. 3 Friction measurements. Representative friction curves for (a) BM, commercial medium with commercial $2.7 \mathrm{~nm}$ COC (CMC), commercial medium with commercial $\sim 2.7 \mathrm{~nm} \mathrm{COC}$ and $\sim 1.0 \mathrm{~nm}$ PFPE lube (CMCL), BM coated with $1.2 \pm 0.2 \mathrm{~nm}$ PFPE lube (BML). b HDM coated with 1-4LG. c HDM with FCVA-ta-C of different thicknesses with 0.3 (3CF), 0.6 (6CF), 1.2 (12CF), and $1.8 \mathrm{~nm}$ (18CF) FCVA-deposited ta-C. d HDM coated with 1-4LG and $1.2 \pm 0.2 \mathrm{~nm}$ PFPE lubricant (L).

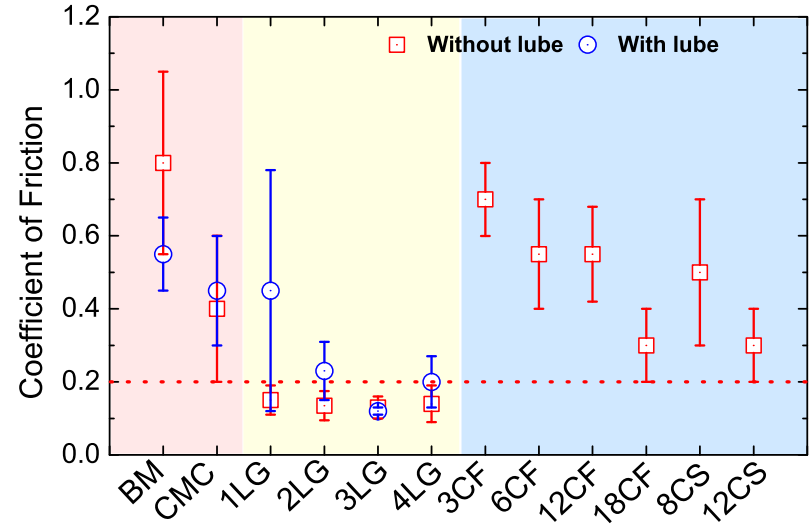

Fig. 4 Comparison of measured coefficients of friction. Average COF from measurements in Fig. 3, with and without lubricant, for 1-4LG and all COC reference samples. The red shaded region highlights the bare media and media with a commercial overcoat, the yellow region highlights media with graphene overcoat, and the blue region highlights media with FCVA-ta-C. The horizontal red dashed line is a reference at $\mathrm{COF}=0.2$. The error bars indicate the spread around the average COF.

(without lube) is $\sim 4$ times lower than $\mathrm{BM}$ and $\sim 2$ times lower than CMC, despite a reduction of thicknesses $\sim 7$ times (for $1 \mathrm{LG}$ ) to 2 times (for 4LG), using the theoretical 1LG thickness, with respect to CMC. Figures $5 \mathrm{~b}, \mathrm{~d}$ and $6 \mathrm{~b}, \mathrm{~d}$ reveal that the wear track width of 1-4LG-coated samples is $\sim 2-4$ times lower and debris transferred to the ball are smaller than $\mathrm{BM}$ and $\mathrm{CMC}$, indicating higher wear resistance. All FCVA COCs with thicknesses from $\sim 0.3$ to $\sim 1.8 \mathrm{~nm}$ show $\sim 2-5$ times higher COF than 1-4LG-coated samples, with and without lube, apart from $1 \mathrm{LG}$ without lube. Pulsed DC sputtered COCs have $\sim 2-3$ times higher COF than 1-4LG-coated samples.
To further analyze the wear tracks, Auger Electron Spectroscopy (AES) imaging is performed using a JEOL JAMP Auger Microprobe, see Supplementary Note 4 and Supplementary Fig. 1 for details. Before AES, scanning electron microscope images are taken to select the AES locations. The AES images inside and outside the wear tracks show the carbon-containing sites, and indicate that the amount of carbon on the wear track increases with increasing number of graphene layers, N. The Co and $\mathrm{Cr}$ intensities inside the wear tracks are higher for $1 \mathrm{LG}$, and decrease with increasing $\mathrm{N}$, due to the increase in $\mathrm{C}$ and the $<1-3 \mathrm{~nm}$ sampling depth of $\mathrm{AES}^{68}$. The $\mathrm{O}$ signal in the wear track appears due to ambient oxygen as the samples are exposed to air before AES, with some contribution from the HDM oxide.

After ball-on-disk tests, the wear tracks are analyzed by recording Raman spectra across the wear track at different positions. Reference 58 introduced a three-stage model of amorphization. Stage 1: graphene to nanocrystalline graphene. Stage 2: nanocrystalline graphene to low $s p^{3}$ amorphous carbon. Stage 3: low $s p^{3}$ to high $s p^{3}$ amorphous carbon. For all spectra in Figs. 5 and 6 , as the $\mathrm{D}$ peak increases, $\mathrm{D}^{\prime}$ and $\mathrm{D}+\mathrm{D}^{\prime}$ appear, whereas the $I(2 \mathrm{D})$ weakens when approaching the wear track, indicating an increase in disorder according to stage 1 . The broad peak between 500 and $1000 \mathrm{~cm}^{-1}$ is due to the glass substrate ${ }^{69}$. At the center of the wear track, all second-order Raman features (i.e., $2 \mathrm{D}, 2 \mathrm{D}^{\prime}$, and $\mathrm{D}+\mathrm{D}^{\prime}$ ) merge, while $I(\mathrm{D})$ decreases and $\mathrm{D}, \mathrm{G}$ become broader, indicating an increase of disorder ${ }^{58}$. 2-4LG is less damaged than 1LG, as for the spectra in Fig. 5a, c and $I(\mathrm{D}) / I(\mathrm{G})$ in Fig. 7a, from at least 5 positions as a function of $\mathrm{COF}$, without and with lubricant (L). Overall, there seems to be no significant trend of $I(\mathrm{D}) / I(\mathrm{G})$ with COF. However, a difference is seen without lubricant: $\mathrm{I}(\mathrm{D}) / \mathrm{I}(\mathrm{G})$ is $\sim 0.95$ and $\sim 1$ for $3-4 \mathrm{LG}$, while for $1-2 \mathrm{LG}$ it is $\sim 1.33$ and $\sim 1.25$. Thus, there are less defects in 3-4LG compared to $1-2 \mathrm{LG}$. The peaks $<1000 \mathrm{~cm}^{-1}$ in Fig. $7 \mathrm{~b}$ are due to the HDM: those $\sim 470 \mathrm{~cm}^{-1}\left(\mathrm{E}_{g}\right), 513 \mathrm{~cm}^{-1}\left(\mathrm{~F}_{2 g}\right), 606 \mathrm{~cm}^{-1}\left(\mathrm{~F}_{2 g}\right), 673 \mathrm{~cm}^{-1}$ 

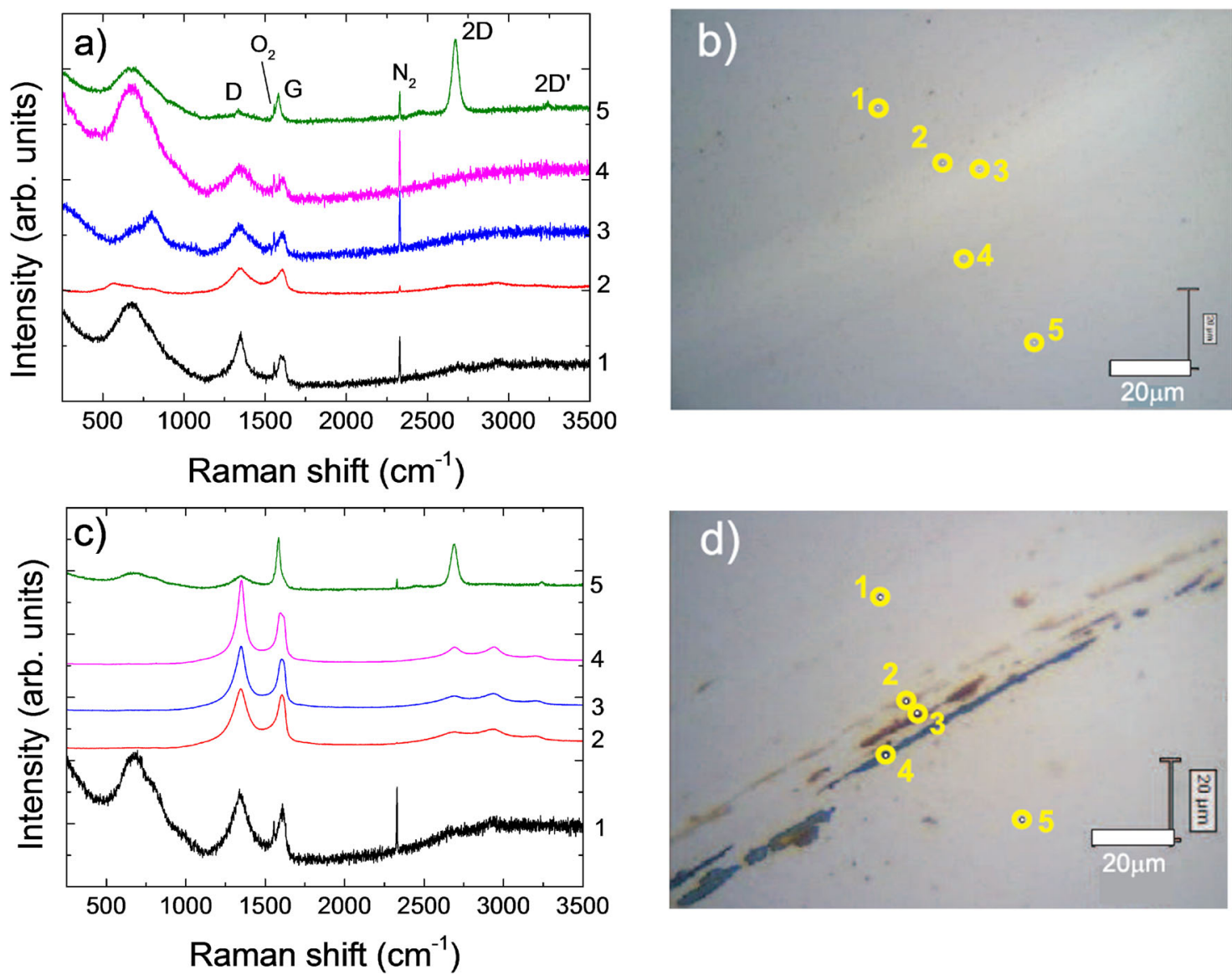

Fig. 5 Raman measurements across wear tracks. Raman spectra, normalized to $/(G)$, recorded across wear tracks of (a) $1 \mathrm{LG}$ and (c) $2 \mathrm{LG}$ and corresponding optical pictures in (b) and (d). In (b), (d) The Raman spectra are recorded across the wear tracks at the positions indicated by the yellow circles to compare graphene before and after friction and wear tests. The colors of the traces are due to a mixture of graphene, HDM components, and polymer residues coming from graphene transfer.

$\left(\mathrm{A}_{1 g}\right)$ are from $\mathrm{Co}_{3} \mathrm{O}_{4}{ }^{70,71}$, while the $\mathrm{A}_{g}$ mode $\sim 550 \mathrm{~cm}^{-1}$ is from $\mathrm{Cr}_{2} \mathrm{O}_{3}{ }^{72}$.

Effect of lubricant. Current commercial HDDs use a layer of perfluoropolyether (PFPE) lubricant (lube) ${ }^{11}$ on top of COCs to further reduce friction and wear, and minimize surface energy. Given the lubricating and corrosion protection properties of $1 \mathrm{LG}$ itself, lube may not be needed for 1LG. However, exploring the compatibility of $1 \mathrm{LG}$ with lube is useful from the fundamental viewpoint.

Friction measurements on lube-coated samples (Fig. 3) show that in certain cases the COF is higher with $\mathrm{L}$, than without. Taking the error bars into account, a significant difference in COF is only observed for 1LG and 1LGL. 1LG has wetting transparency ${ }^{73}$, i.e., it is thin enough that its introduction does not affect the wettability of an underlying substrate. Without overcoat, the L containing HDM, as in BML, shows very high $\mathrm{COF} \sim 0.55$ and wear, due to the metal (medium) L-induced catalysis $^{74}$. 1LG is not enough to avoid the interaction of $\mathrm{L}$ with medium, hence higher COF $\sim 0.45$ and wear is observed in 1LGL. Without L, $1 \mathrm{LG}$ shows very low $\mathrm{COF} \sim 0.15$ and wear. A minor difference in case of $2 \mathrm{LG}$ versus $2 \mathrm{LGL}$ can be seen. However, for $3 \mathrm{LG}$ versus $3 \mathrm{LGL}$ and $4 \mathrm{LG}$ versus $4 \mathrm{LGL}$ the difference in average $\mathrm{COF}$ is marginal. L on 1LG results in higher and inconsistent $\mathrm{COF}$ and wear, as some measurements show lower $\mathrm{COF} \sim 0.15$, some higher $\sim 0.78$, with $\mathrm{COF}$ increasing after few thousands cycles. The COF of $1 \mathrm{LG}$, on an average, is $\sim 0.45$, i.e., $\sim 3$ times its non-lubricated counterpart. Thus, COFs of $2-4 \mathrm{LG}$ are more or less similar to those of the non-lubricated counterparts, suggesting that $2-4 \mathrm{LG}$ without $\mathrm{L}$ are lubricious enough, and that $\mathrm{L}$ does not improve lubricity. The D and G peaks in the Raman measurements in Figs. 5 and 6 on 1-4LG confirm the presence of carbon on the wear tracks, and transfer of debris to the balls, similar to the non-lubricated counterparts.

Corrosion. Co-alloys have a great propensity to corrode, mainly due to Co oxidation ${ }^{75}$. This results in the loss of magnetic properties ${ }^{75}$, hence this is one of the major concerns for the longterm functionality and durability of HDDs ${ }^{14,51,75}$. To examine the corrosion protection efficiency of $1-4 \mathrm{LG}$ and compare their performance with state-of-the-art COCs, the corrosion of different uncoated- and coated-HDM, exposed to an electrolyte solution $\sim 0.1 \mathrm{M} \mathrm{NaCl}$ similar to that used in refs. ${ }^{75}$ on a $\sim 0.24 \mathrm{~cm}^{2}$ area, is investigated using an electrochemical corrosion method $^{51,75}$. The measurements are performed with a 3 -electrode setup with a Pt wire as counter electrode, $\mathrm{Ag} / \mathrm{AgCl}$ as reference electrode and HDM as working electrode, to which the potential is applied ${ }^{51}$. Each test consists of anodic and cathodic sweeps, where the potential is varied and the corresponding current measured ${ }^{51}$. Every sweep is conducted at different locations, with at least 3 sets of 6 sweeps on each sample. The so-called Tafel's analysis ${ }^{75}$ is done by plotting anodic and cathodic curves on a semi-logarithmic scale of potential versus log current. The linear part of the logarithmic anodic and cathodic currents are 

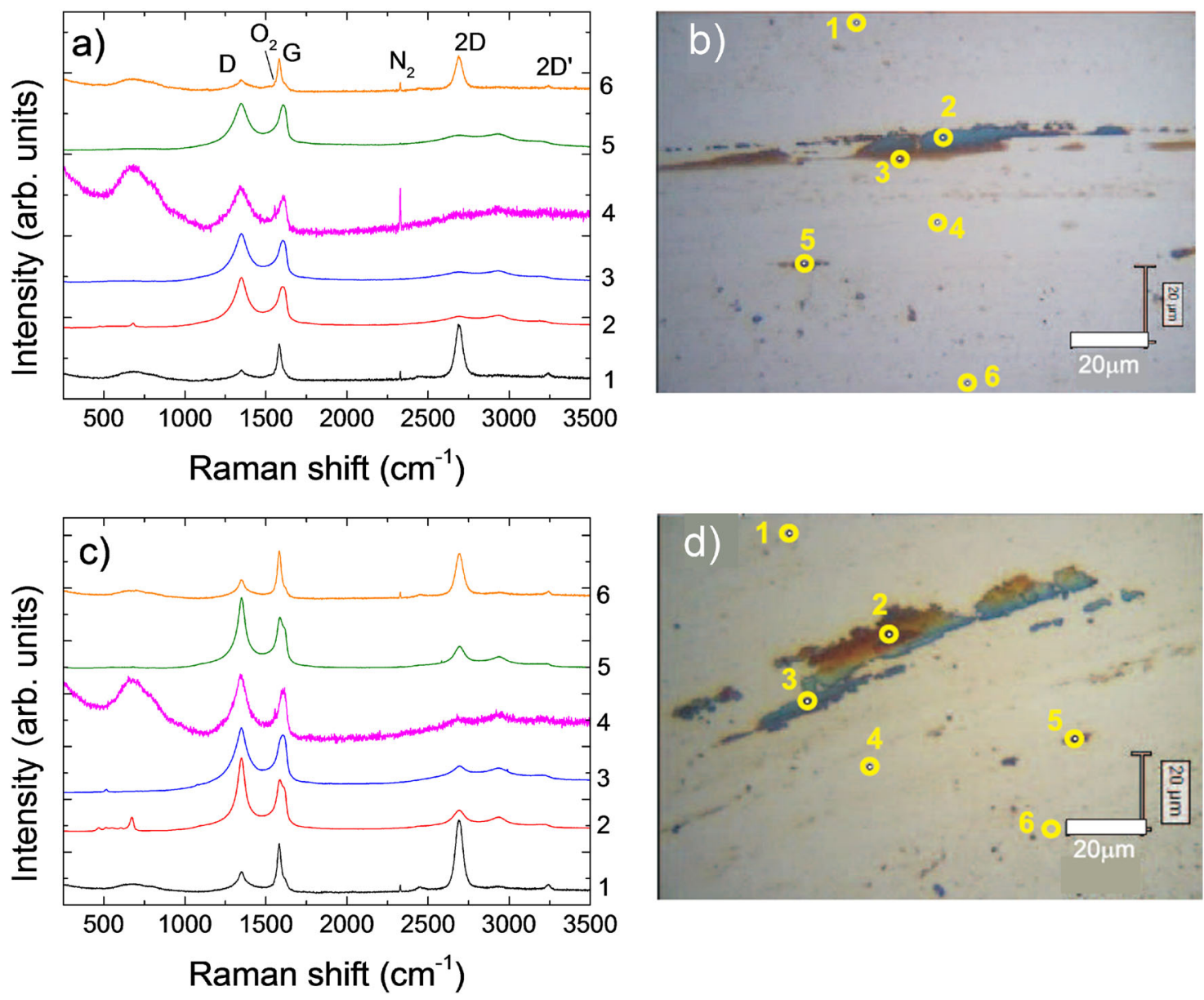

Fig. 6 Raman measurements across wear tracks. Raman spectra, normalized to I(G), recorded across wear tracks of (a) $3 L G$ and (c) $4 L G$ and corresponding optical pictures in (b) and (d). In (b), (d) The Raman spectra are recorded across the wear tracks at the positions indicated by the yellow circles to compare graphene before and after friction and wear tests. The colors of the traces are due to a mixture of graphene, HDM components, and polymer residues coming from graphene transfer.

extrapolated ${ }^{75}$, and the intercept of these lines gives the corrosion current ${ }^{75}$, and the corrosion current density $J_{\text {corr }}$ when divided by the contact area.

The corrosion protection efficiency (CPE) defines how efficient the $\mathrm{COC}$ is in protecting against HDM corrosion. This is defined as ${ }^{51}$ :

$$
\mathrm{CPE}(\%)=\frac{J_{\text {corr }}^{0}-J_{\text {corr }}}{J_{\text {corr }}^{0}} \times 100,
$$

where $J_{\text {corr }}^{0}$ is the BM corrosion current density and $J_{\text {corr }}$ that of coated HDM.

When a metal is exposed to a corrosive solution, it releases ions that leave behind electrons, which can be observed in an anodic reaction as ${ }^{75}$ :

$$
\mathrm{M} \rightarrow \mathrm{M}^{n+}+n e^{-},
$$

where $\mathrm{M}$ represents the metal and $n$ the number of electrons released by it. For Co-alloy-based systems, the metal dissolution, which decreases the anode conductivity ${ }^{75}$, can be written as ${ }^{75}$ :

$$
\mathrm{Co} \rightarrow \mathrm{Co}^{2+}+2 e^{-},
$$

As the corrosion reaction involves the transfer of electrons and ions between metal and solution ${ }^{75}$, the corrosion rate varies with corrosion current ${ }^{75}$, hence $J_{\text {corr }}$ varies inversely with corrosion resistance, i.e., the COC ability to reduce the HDM corrosion ${ }^{51,75}$.
The BM shows the highest $J_{\text {corr }}$, indicating the greatest propensity to corrode (Fig. 8). Introduction of 1-4LG reduces HDM corrosion. $J_{\text {corr }}$ decreases with $N$, from $\sim 5.3 \mathrm{nA} / \mathrm{cm}^{2}$ for 2LG to $\sim 4.4 \mathrm{nA} / \mathrm{cm}^{2}$ for $4 \mathrm{LG}$, Fig. 8 , and CPE increases. The COC defects and pinholes are the active corrosion sites ${ }^{75}$, and their electrical conductivity could further add galvanic corrosion ${ }^{75}$. The COC should be defect- and pinhole-free, smooth, and with excellent barrier properties to minimize HDM oxidation and corrosion. Reference ${ }^{43}$ showed that $1 \mathrm{LG}$ can be used as a corrosion barrier for metallic surfaces, and suggested that this should be uniform and defect-free to achieve corrosion protection. In our case, $1 \mathrm{LG}$ reduces $J_{\text {corr }}$ by $\sim 2.5$ times with respect to $\mathrm{BM}$, but it is twice that of BM with $\sim 2.7 \mathrm{~nm}$ COC. For $2 \mathrm{LG}, J_{\text {corr }}$ is $\sim 3.8$ times smaller than $\mathrm{BM}$, and marginally higher than COC. This is remarkable as the $2 \mathrm{LG}$ thickness $\sim 0.7 \mathrm{~nm}$, is $\sim 4$ times lower than $2.7-\mathrm{nm}$-thick COC. Beyond 2LG, $J_{\text {corr }}$ and CPE remain similar, adding marginal anti-corrosion improvement (Fig. 8). The higher corrosion protection in 2-4LG with respect to $1 \mathrm{LG}$ is mainly due to the fact that the increase in $\mathrm{N}$ improves the HDM coverage, leading to the reduction of active corrosion sites. 2-4LG display corrosion protection similar to COC, attributed to better barrier properties. Figure 8 indicates that, for a similar thickness, graphene-based overcoats have lower $J_{\text {corr }}$ than amorphous COCs, indicating greater protection. Figure 8 shows that the corrosion resistance performance of $1 \mathrm{LG}$ is $\sim 2.5$ times better than BM, but $\sim 2$ times higher than COCs. By adding graphene layers the corrosion resistance is improved. The 

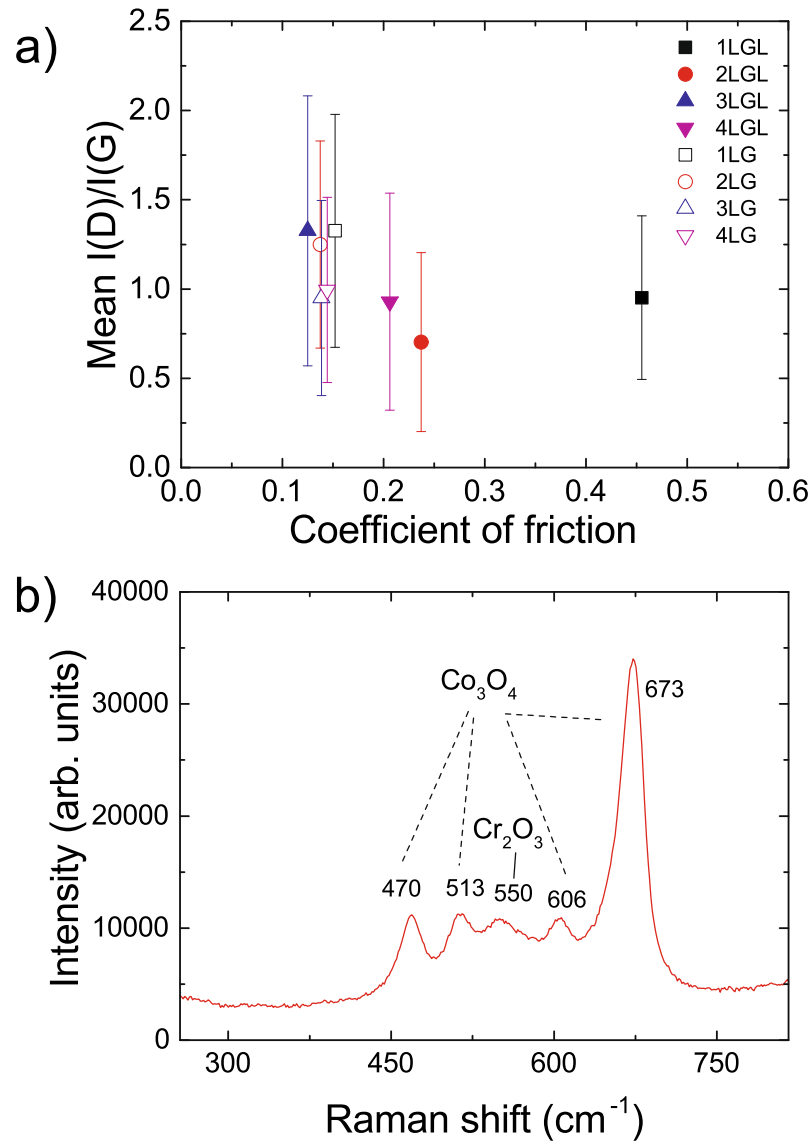

Fig. 7 Raman analysis of defect-induced peaks and hard disk media. a Mean I(D)/I(G) of 1-4LG-coated samples, with and without lube, after friction tests, as a function of COF. The error bars come from averaging over the intensity ratios of the peaks in the Raman spectra recorded across the wear tracks. $\mathbf{b}$ Raman spectrum on a wear track. The region $\sim 400-750 \mathrm{~cm}^{-1}$ shows peaks of the underlying substrate.

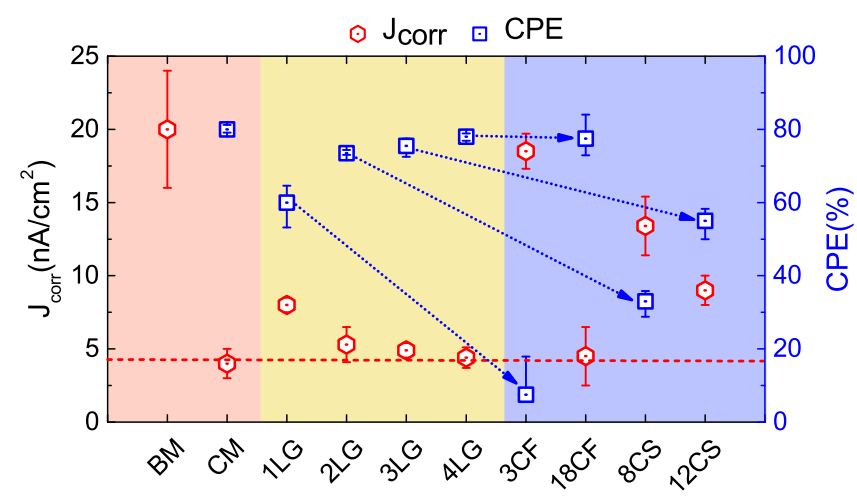

Fig. 8 Corrosion measurements. Corrosion current density $J_{\text {corr }}$ and corrosion protection efficiency (CPE) for different samples. The corrosion resistance varies inversely with $J_{\text {corr }} 51.2-4 L G$ have similar CPE to $\sim 2.7 \mathrm{~nm}$ CMC and $1.8 \mathrm{~nm} \mathrm{COC}$ in sample 18CF. The dotted line is a guide to the eye for $J_{\text {corr }}$ of different samples. The arrow compares various 1-4LG-COCs pairs, such as $1 \mathrm{LG} \rightarrow 3 \mathrm{CF}, 2 \mathrm{LG} \rightarrow 8 \mathrm{CS}, 3 \mathrm{LG} \rightarrow 12 \mathrm{CS}$, and $4 \mathrm{LG} \rightarrow 18 \mathrm{CF}$, as the 1-4LGbased sample in each pair is always slightly thinner than the amorphous COC. The red shaded region highlights the bare media and media with a commercial overcoat, the yellow region highlights media with graphene overcoats and the blue region highlights media with FCVA-deposited ta-C. The horizontal red dashed line is a reference at $J_{\text {corr }}=4 \mathrm{nA} / \mathrm{cm}^{2}$. The error bars stem from taking the average of multiple repeated measurements. difference in corrosion current density between COC and 2-4LG is $30-10 \%$. Thus, the corrosion resistance of $2-4 \mathrm{LG}$ approaches that of COCs. Taking into account that the theoretical thickness of $1 \mathrm{LG}$ is $\sim 8-9$ times lower than state of the art COCs, and that a COC having the same thickness as $1 \mathrm{LG}$ would provide no protection and be full of holes ${ }^{19}, 1 \mathrm{LG}$ comfortably beats any COC of the same thickness (i.e., same storage density).

\section{Discussion}

Friction is defined as resistance to sliding ${ }^{36,76}$. At the macroscopic level, Amonton's law ${ }^{76}$ states that the frictional force between two bodies varies proportionally to the normal force. Hence:

$$
\mathrm{COF}=\frac{F}{W},
$$

where $F[\mathrm{mN}]$ is the frictional force and $W[\mathrm{mN}]$ is the normal force. This does not take into account the area of contact at the microscopic level ${ }^{76}$. Reference ${ }^{76}$ suggested that the contact between two bodies contains several smaller contacts, called asperities, with the sum of the areas of these asperities being lower than the apparent macroscopic area ${ }^{76}$. Thus, from ref. ${ }^{76}, F$ in micro-tribology can be expressed as:

$$
F=\tau \sum A_{\text {asp }},
$$

where $\tau$ is the shear strength, i.e., the stress required to shear the contacting interfaces and enable sliding, expressed as shear force/ area and $\sum A_{\text {asp }}$ is the sum of the areas of the asperities, also called real contact area ${ }^{76}$. Hence, friction also depends on $\sum A_{\text {asp. }}$. Reference $^{76}$ showed that the stresses over regions of contact reach the elastic limit of the material and cause plastic deformation. Thus, the average pressure $(p)$ in a contact region is governed by the normal force, and is given by $p=W / A^{77}$. The zone of deformation increases with increase in load, and $p$ on the region of contact tends to a stable value, which eventually causes the deformation to become entirely plastic. The process continues until the area of contact becomes sufficient to support the load $W$. Consequently, the normal force can be expressed as:

$$
W=p \sum A_{\text {asp }},
$$

Thus, Eqs. (4)-(6), give:

$$
\mathrm{COF}=\frac{F}{W}=\frac{\tau}{p}
$$

A co-alloy is mechanically softer ${ }^{78}$ than $\mathrm{Al}_{2} \mathrm{O}_{3}{ }^{79}$. When a metallic co-alloy surface slides against $\mathrm{Al}_{2} \mathrm{O}_{3}$, it causes the HDM surface to undergo plastic deformation ${ }^{76}$, generating wear and high COF $\sim 0.8$, Figs. 3 and 4 , consistent with the friction results for metallic surfaces in ref. ${ }^{76}$. This could be due to the formation of an adhesive contact at the HDM-ball interface, due to the high surface energy of Co-alloy-based $\mathrm{HDM} \sim 42.8 \mathrm{mN} / \mathrm{m}^{80}$, and the presence of contaminants at the interface that may enhance the interaction between the two bodies ${ }^{81}$. When $1 \mathrm{LG}$ is placed on HDM, the COF decreases $\sim 4$ times and negligibly changes with $N$.

At the nanoscale, the friction of $1 \mathrm{LG}$ on $\mathrm{Si} / \mathrm{SiO}_{2}$ against $\mathrm{Si}$ tips, measured at a low load $\sim 1 \mathrm{nN}$ and scan speed $\sim 1-10 \mu \mathrm{m} / \mathrm{s}$, was explained based on out-of-plane deformation in front of a scanning probe tip, the so-called puckering effect ${ }^{39,82}$. This enhances the contact area, hence friction. The out-of-plane deformation is suppressed with increasing $\mathrm{N}^{82}$, resulting in reduced COF. This is not applicable to ball-on-disk tribological tests of 1-4LG, due to the significantly larger dimension (ball radius $\sim 2.0 \pm 0.1 \mathrm{~mm}$ ) of the counterface, much higher load $\sim 20 \mathrm{mN}$ and higher speed $\sim 100 \mathrm{rpm}$ or $1.05 \mathrm{~cm} / \mathrm{s}$, which do not precisely differentiate the frictional characteristics of 1-4LG. Thus, all coated media show similar friction (Fig. 3b). Since the layers are coupled by van-derWaals forces, they shear easily, as their interfacial shear strength 
is low, with a shear force per unit area $\alpha \sim 12.8 \cdot 10^{18} \mathrm{~N} / \mathrm{m}^{383}$ and a resulting shear modulus $C_{44} \sim 4.3 \mathrm{GPa}^{83}$. The ease of shearing at sliding contacts results in smoother frictional curves (and marginally lower COF) in 2-4LG with respect to $1 \mathrm{LG}$. The improved wear resistance for 1-4LG-coated HDM is attributed to reduced friction, wear, and excellent mechanical properties of 1LG: breaking strength $\sim 42 \mathrm{~N} / \mathrm{m}^{84}$, Young's Modulus $\sim 1 \mathrm{TPa}^{84}$, flexibility (1LG can be stretched up to $\sim 20 \%$ without breaking ${ }^{84}$ ).

When compared to $\mathrm{BM}$, the COF reduction of 1-4LG-coated HDM could be attributed to reduced adhesive interaction (lower surface energy) ${ }^{36,85}$, as well as incommensurability of the lattice planes sliding against each other at the tribological interface ${ }^{35}$, occurring when the hills of a surface with lattice spacing $a$ do not match the valleys of another surface of lattice spacing $b$, such that $b / a$ is an irrational number ${ }^{35}$. This is consistent with what suggested in ref. ${ }^{35}$ for $3-4 \mathrm{LG}$ on $\mathrm{Si} / \mathrm{SiO}_{2}$ sliding against an a-C:Hcoated steel ball, and for 1-3LG on a steel sliding against a steel ball ${ }^{35,37}$ in macroscale ball-on-disk conditions, e.g., with a normal force in the $z$ direction $N_{z} \sim 0.5-3 \mathrm{~N}$, and speeds $\sim 0.6-25 \mathrm{~cm} / \mathrm{s}$.

The AES on the wear tracks and Raman measurements across the wear tracks, after ball-on-disk tribological tests, in Figs. 5 and 6 show that a C signal is still present in all 1-4LG samples, even though disorder-induced peaks appear. Reference ${ }^{86}$ also found C on wear tracks after microscale friction and wear tests of graphene on $\mathrm{SiC}$. The counterface also reveals transferred debris, consisting of disordered carbon and underlying substrate atoms. This implies that, when a $20 \mathrm{mN}$ load is applied and the sample starts to rotate at $\sim 2.1 \mathrm{~cm} / \mathrm{s}, 1 \mathrm{LG}$ could be turned into patches, due to the large contact load. Reference ${ }^{86}$ reported transformation of continuous layers of 1-2LG into 1-2LG patches during microscale tribology, despite using lower load $\sim 0.1-1 \mathrm{mN}$ and speeds $\sim 30-50 \mu \mathrm{m} / \mathrm{s}$ compared to us. This led to a distribution of 1LG patches along the wear track, and transfer of 1LG-containing debris on the counterface. This also happens for 2-4LG-coated HDM. Raman measurements in Figs. 5 and 6 show disordered carbon on the wear tracks, with disorder lower than for 1LG, as revealed by $I(\mathrm{D}) / I(\mathrm{G})$ (Fig. 7). This implies that $1 \mathrm{LG}$ acts as lubricant when in contact with another 1LG, and is not completely removed during tribological tests. AES also reveals a progressive increase in $\mathrm{C}$ and $\mathrm{C}$-containing sites on the wear tracks with increasing number of layers. The debris transferred to the ball also contain $\mathrm{C}$. Therefore, the formation of disordered carbon debris on both surfaces facilitates smooth sliding, and contributes to maintaining the low COF. The marginally lower friction in 2-4LG as compared to $1 \mathrm{LG}$ can be linked to $I(\mathrm{D}) / I(\mathrm{G})$ (Fig. 7a), with lower disorder corresponding to lower friction, although this does not apply for 1-4LGL.

Figure 9 is the proposed mechanism for friction reduction for 1LG and 3LG-coated HDM, where C debris are on both ball and wear track. To validate these assumptions, we perform molecular dynamics (MD) simulations of a Co|Pt-sapphire system with and without 1 and 4LG, as discussed in Supplementary Note 8.

The COF is estimated as the ratio of sliding and normal force, block averaged every 25 ps. Figure 10a-e compares the computed $\mathrm{COF}$ and surface disorder of $\mathrm{Co} \mid \mathrm{Pt}, 1 \mathrm{LG}$, and $4 \mathrm{LG}$. The surface disorder represents the friction-induced damage/wear of the HDM, and its value is derived considering $0 \%$ surface disorder before friction measurements. The amount of disorder in HDM is quantified using the centrosymmetry parameter, a measure of the local lattice disorder around an atom. This is 0 for a perfect lattice, whereas when a point defect exists, i.e., when the symmetry is broken, it assumes a larger positive value, see Supplementary Note 8 for details. The simulations indicate that BM develops substrate disorder up to $\sim 14.5 \%$ within 350 ps (Fig. 10b, e).

High wear is also observed for BM in the experiments in Fig. 3a. An additional 250 ps where the forces on atoms are also collected are used to obtain the COF (Supplementary Fig. 5). The COF averaged over these $250 \mathrm{ps}$ is $\sim 0.82 \pm 0.1$. $1 \mathrm{LG}$ reduces the average simulated COF to $\sim 0.18 \pm 0.02$. Figure $10 \mathrm{c}$ shows that $1 \mathrm{LG}$ maintains its structural integrity with fraction of disorder $\sim 1 \%$ on

a)
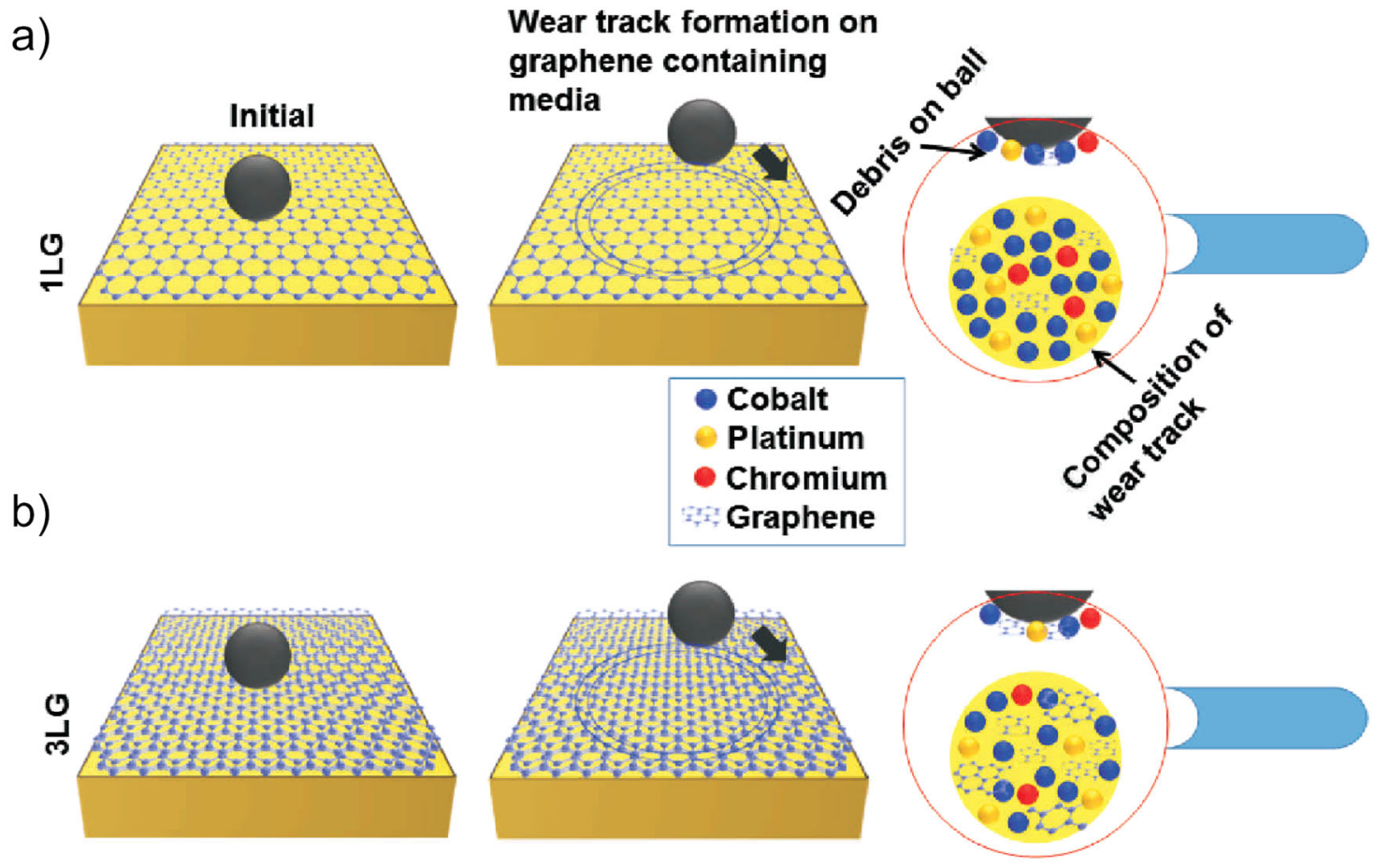

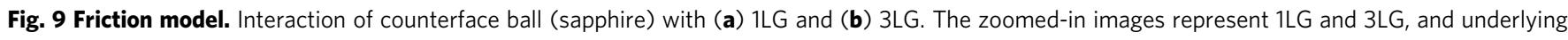
substrate atoms (Co, Pt, $\mathrm{Cr}$, etc.) on counterface ball and wear track, where the amount of $\mathrm{C}$ debris increases with $\mathrm{N}$. The debris generated on both interacting bodies maintain the lower COF of 1-4LG with respect to BM. 

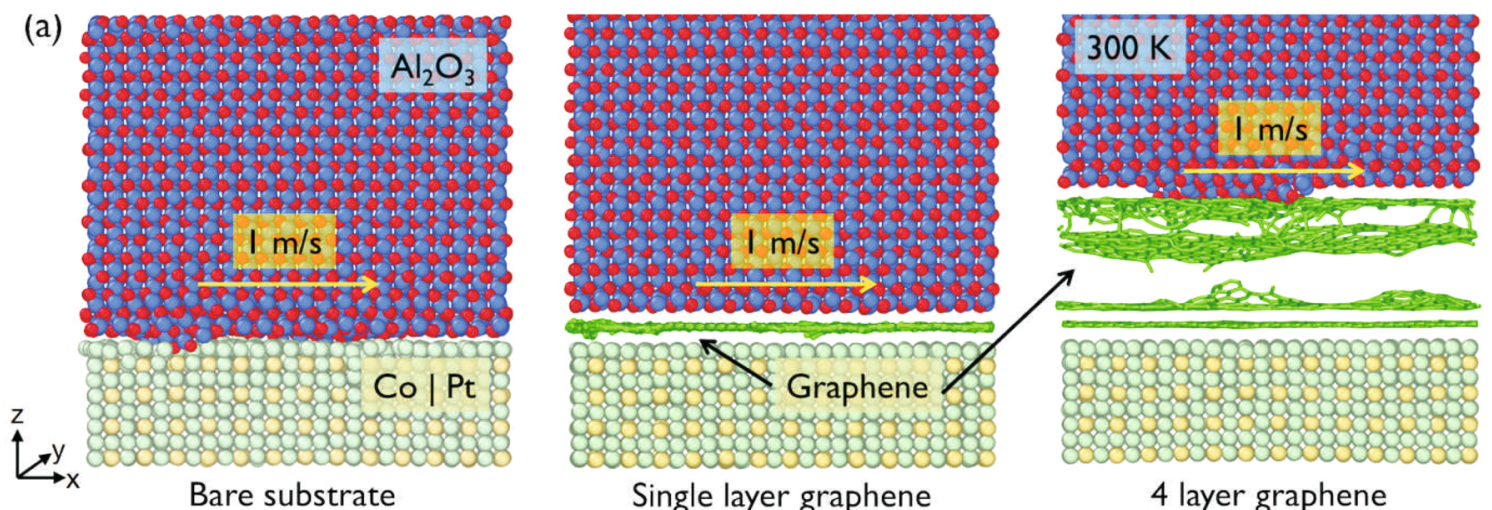

Single layer graphene
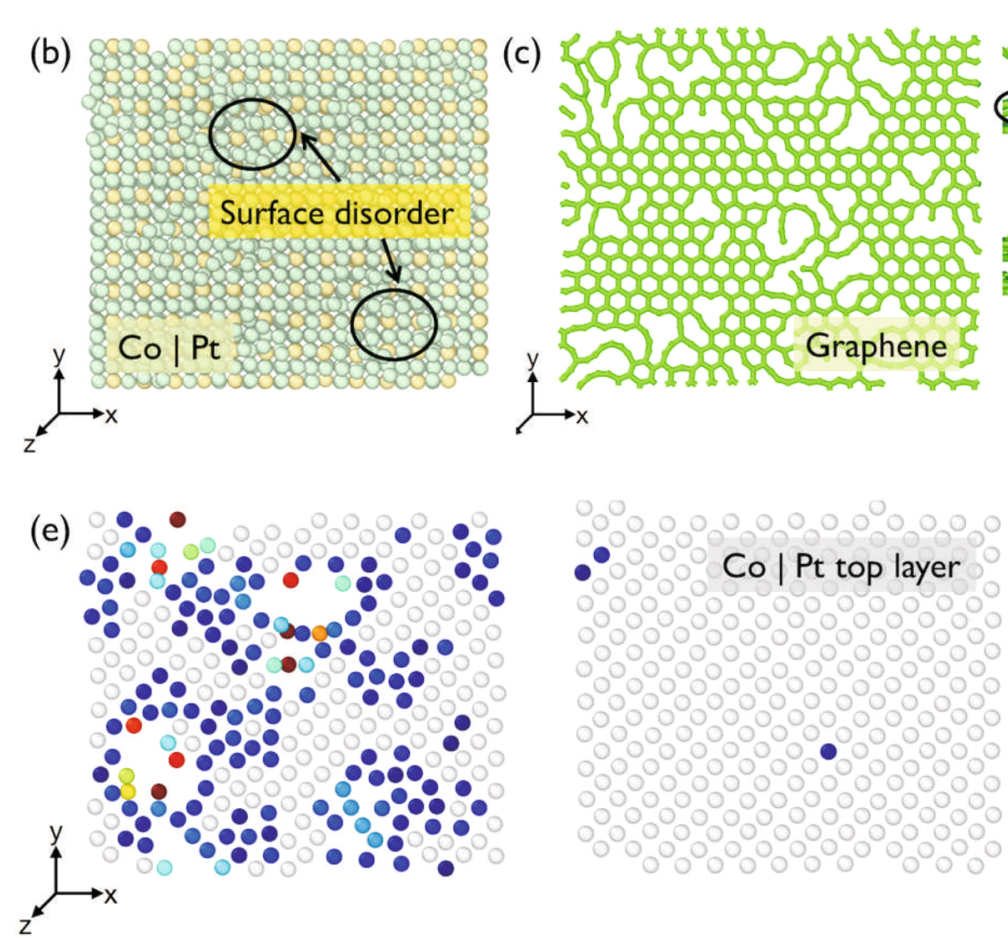

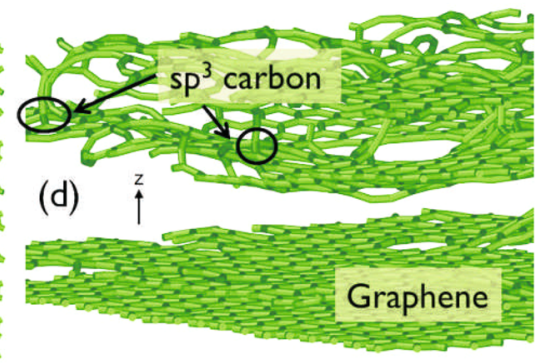

Centrosymmetry

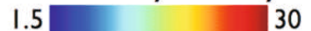

Fig. 10 Simulations of friction process. Light green spheres indicate $\mathrm{Co} \mid \mathrm{Pt}$, red and blue spheres indicate $\mathrm{Al}_{2} \mathrm{O}_{3}$. a Simulation setup for evaluating wear between $\mathrm{Al}_{2} \mathrm{O}_{3}$ and $\mathrm{Co}$ Pt. Simulations are performed for $\mathrm{BM}, 1,4 \mathrm{LG}$ at $300 \mathrm{~K}$, with materials sliding against each other at $1 \mathrm{~m} / \mathrm{s}$. $\mathbf{b} \mathrm{Interfacial}$ surface atoms of bare ColPt showing disorder. Two representative disorder regions are indicated by circles. c Structure of defected 1LG. d Structure of $4 \mathrm{LG}$. The top two layers are attracted towards the $\mathrm{Al}_{2} \mathrm{O}_{3}$ block with the formation of $s p^{3}$ cross-links. e Disorder of interfacial Co|Pt surface using the centrosymmetry parameter.

average, as a consequence of the low COF between the blocks, indicating higher resistance to wear as compared to BM. 4LG further improves the tribological behavior. The COF drops slightly to $\sim 0.14 \pm 0.0 .02$ and substrate disorder is reduced to $\sim 0.1 \%$, much lower than in BM, suggesting that the BM surface remains mostly unaffected. Simulations suggest partial transfer of graphene patches to the sapphire ball. The Raman analysis of wear tracks in Figs. 5 and 6 shows tribo-induced disorder in 1-4LG transfer of C to the ball. Thus, the patches containing debris on both surfaces are responsible for the lower COF in 1-4LG-coated HDM, maintaining higher wear resistance than BM.

Reference 87 showed that potential corrugations on sliding surfaces can impact friction force and anisotropy of MLG. It reported that higher friction forces result from larger corrugations of the potential energy and depend on $N$. This is consistent with our experiments in Figs. 3 and 4, and simulations in Fig. 10.
In summary, we analyzed 1-4LG-coated media and found that they can overcome the tribological and corrosion issues of current Co-alloy-based HDM, with laser irradiation stability on FePtbased HDM for HAMR. The overall performance of graphenecoated media exceeds that of thicker commercial COC, as well as other amorphous carbons of comparable/higher thicknesses prepared by FCVA and sputtering. Given the tribological, corrosion, and thermal stability characteristics coupled with an AFM thickness $\sim 4$ times (for 1LG) to $\sim 1.3$ times (for $4 \mathrm{LG}$ ) lower than stateof-the-art 2.7-nm COCs, we expect 1-4LG-based overcoats to meet the requirements for $4-10 \mathrm{~Tb} / \mathrm{in}^{2}$ areal density HDDs, when employing suitable recording technologies, such as HAMR and HAMR+BPM. Graphene-coated media have better corrosion and wear resistance with a $\mathrm{COF}<0.2$. A single layer of graphene is enough to reduce corrosion $\sim 2.5$ times and can withstand HAMR conditions. Our results imply that $>2$ LG-based coatings could be 
used as a tribological interface for various other materials/devices, such as micro- and nano-electromechanical systems.

\section{Methods}

Graphene growth and transfer. 1LG is grown by CVD on a $35 \mu \mathrm{m} \mathrm{Cu}$ foil. The substrate is loaded into a hot wall tube furnace evacuated to $\sim 1 \mathrm{mTorr}$. The Cu foil is annealed in hydrogen at $1000{ }^{\circ} \mathrm{C}$ for $30 \mathrm{~min}$. This reduces the copper oxide surface and increases the $\mathrm{Cu}$ grain size. The growth process starts when $5 \mathrm{sccm}$ $\mathrm{CH}_{4}$ is added to $\mathrm{H}_{2}$. After 30 min the substrate is cooled down for several hours in vacuum ( 1 mTorr) to room temperature and unloaded.

We use a polymethyl methacrylate (PMMA)-based wet transfer ${ }^{52,53}$. First, $\sim 500$ nm PMMA is spin-coated on the sample. The PMMA/1LG/Cu stack is then placed in an aqueous solution of ammonium persulfate to etch $\mathrm{Cu}^{52}$. When $\mathrm{Cu}$ is fully etched, the graphene/PMMA-stack is placed into a de-ionized (DI) water bath to rinse any acid residuals and subsequently fished out using the HDM substrate. After drying for one day at room temperature, the sample is placed in acetone to remove PMMA, leaving 1LG on the HDM. By repeating the steps described above, several 1LGs are transferred to create an MLG stack. The same procedure is used to place $1 \mathrm{LG}$ and MLG onto FePt.

\section{Data availability}

All relevant data are available from the authors upon reasonable requests. Unique identifiers such as DOI and hyperlinks for any other data sets are also available from the authors upon reasonable request. Source data are provided with this paper.

\section{Code availability}

All relevant custom software is available from the authors upon reasonable request

Received: 2 June 2019; Accepted: 17 March 2021;

Published online: 17 May 2021

\section{References}

1. Reinsel, D., Gantz, J. \& Rydning, J. Data age 2025: The Evolution of Data to Life-Critical. IDC White Paper 1-25 (2017)

2. Furrer, S. et al. $201 \mathrm{~Gb} / \mathrm{in}^{2}$ Recording areal density on sputtered magnetic tape. In Digest of 28th Magnetic Recording Conference 97-98 (2017).

3. Grochowski, E. \& Goglia, P. Magnetic hard disk drive today's technical status and future. https://www.snia.org/sites/default/files/SDC/2016/presentations/ keynote_general/Grochowski-Goglia_Magnetic_Hard_Disk_Drive.pdf. (2016).

4. Statista Research Department. WW HDD market declining to 395 million units shipped in 2017-statista. Storage Newsletter. https://www. storagenewsletter.com/2017/10/10/ww-hdd-market-declining-to-395-millionunits/-shipped-in-2017-statista/ (2017).

5. Nordrum, A. The fight for the future of the disk drive. IEEE Spectrum 56, 44-47 (2018)

6. Marchon, B. et al. The head-disk interface roadmap to an areal density of 4 Tbit/in². Adv. Tribol. 2013, 521086 (2013).

7. Casiraghi, C., Ferrari, A. C., Ohr, R., Chu, D. \& Robertson, J. Surface properties of ultra-thin tetrahedral amorphous carbon films for magnetic storage technology. J. Diam. Rel. Mats. 13, 1416-1421 (2004).

8. Casiraghi, C. et al. Ultra-thin carbon layer for high density magnetic storage devices. Diam. Rel. Mats. 13, 1480-1485 (2004).

9. Piazza, F. et al. Large area deposition of hydrogenated amorphous carbon films for optical storage disks. Diam. Rel. Mats. 13, 1505-1510 (2004).

10. Piazza, F. et al. Protective diamond-like carbon coatings for future optical storage disks. Diam. Rel. Mats. 14, 994-999 (2005).

11. Dwivedi, N. et al. Ultrathin carbon with interspersed graphene/fullerene-like nanostructures: a durable protective overcoat for high density magnetic storage. Sci. Rep. 5, 11607 (2015)

12. Rajauria, S., Canchi, S. V., Schreck, E. \& Marchon, B. Nanoscale wear and kinetic friction between atomically smooth surfaces sliding at high speeds. Appl. Phys. Lett. 106, 081604 (2015).

13. Ferrari, A. C. Diamond-like carbon for magnetic storage disks. Surf. Coat. Technol. 180, 190-206 (2004).

14. Casiraghi, C., Robertson, J. \& Ferrari, A. C. Diamond-like carbon for data and beer storage. Mater. Today 10, 44-53 (2007).

15. Dwivedi, N. et al. Understanding the role of nitrogen in plasma-assisted surface modification of magnetic recording media with and without ultrathin carbon overcoats. Sci. Rep. 5, 7772 (2015).

16. Casiraghi, C., Ferrari, A. C. \& Robertson, J. The smoothness of tetrahedral amorphous carbon. Diam. Rel. Mats. 14, 913-920 (2005).

17. Moseler, M., Gumbsch, P., Casiraghi, C., Ferrari, A. C. \& Robertson, J. The ultrasmoothness of diamond-like carbon surfaces. Science 309, 1545 (2005).
18. Pisana, S., Casiraghi, C., Ferrari, A. C. \& Robertson, J. Roughness evolution during growth of hydrogenated tetrahedral amorphous carbon. Diam. Rel. Mats. 15, 898-903 (2006).

19. Casiraghi, C. et al. Dynamic roughening of tetrahedral amorphous carbon. Phys. Rev. Lett. 91, 226104 (2003).

20. Piramanayagam, S. N. Perpendicular recording media for hard disk drives. $J$ Appl. Phys. 102, 011301-22 (2007).

21. Plumer, M. L., van Ek, J. \& Cain, W. C. New paradigms in magnetic recording. Phys. Canada 67, 25-29 (2011)

22. Weller, D. et al. FePt heat assisted magnetic recording media. J. Vac. Sci. Technol. B 34, 060801-10 (2016).

23. Weller, D. \& Moser, A. Thermal effect limits in ultrahigh-density magnetic recording. IEEE Trans. Magn. 35, 4423-4439 (1999).

24. Kryder, M. H. et al. Heat assisted magnetic recording. Proc. IEEE 96, 1810-1835 (2008)

25. Challener, W. A. et al. Heat-assisted magnetic recording by a nearfieldtransducer with efficient optical energy transfer. Nat. Photonics 3, 220-224 (2009)

26. Vogler, C. et al. Noise mechanisms of heat-assisted-magnetic recording. J. Appl. Phys. 120, 153901 (2016).

27. Plumer, M. L., van Ek, J. and Weller, D. The Physics Of Ultrahigh Density Magnetic Recording 1-352 (2001).

28. Jones, P. M., Ahner, J., Platt, C. L., Tang, H. \& Hohlfeld, J. Understanding Disk Carbon Loss Kinetics for Heat Assisted Magnetic Recording. IEEE Trans. Magn. 50, 3300704 (2014).

29. Ji, R., Ma, Y., Shakerzadeh, M., Seet, H. L. \& Hu, J. F. Laser irradiation effect on carbon overcoat for HAMR application. Surf. Interface Anal. 46, 204-208 (2014).

30. Mangolini, F., Rose, F., Hilbert, J. \& Carpick, R. W. Thermally induced evolution of hydrogenated amorphous carbon. Appl. Phys. Lett. 103, 161605 (2013)

31. Wang, N., Komvopoulos, K., Rose, F. \& Marchon, B. Structural stability of hydrogenated amorphous carbon overcoats used in heat-assisted magnetic reco rding investigated by rapid thermal annealing. J. Appl. Phys. 113, 083517 (2013).

32. Pathem, B. K. et al. Carbon overcoat oxidation in heat-assisted magnetic recording. IEEE Trans. Magn. 49, 3721-3724 (2014).

33. Ferrari, A. C. et al. Stress reduction and bond stability during thermal annealing of tetrahedral amorphous carbon. J. Appl. Phys. 85, 7191-7197 (1999).

34. Teo, K. B. K. et al. Highest optical gap tetrahedral amorphous carbon. Diam. Rel. Mats. 11, 1086-1090 (2002).

35. Berman, D., Deshmukh, S. A., Subramanian, S. K. R. S., Erdemir, A. \& Sumant, A. V. Friction. Macroscale superlubricity enabled by graphene nanoscroll formation. Science 348, 1118-1122 (2015).

36. Berman, D., Erdemir, A. \& Sumant, A. V. Graphene: a new emerging lubricant. Mater. Today 17, 31-42 (2014).

37. Berman, D., Deshmukh, S. A., Subramanian, S. K. R. S., Erdemir, A. \& Sumant, A. V. Extraordinary macroscale wear resistance of one atom thick graphene layer. Adv. Funct. Mater. 24, 6640-6646 (2014).

38. Berman, D., Erdemir, A. \& Sumant, A. V. Graphene as a protective coating and superior lubricant for electrical contacts. Appl. Phys. Lett. 105, 231907 (2014).

39. Lee, C. et al. Frictional characteristics of atomically thin sheets. Science $\mathbf{3 2 8}$, 76-80 (2010).

40. Chen, $\mathrm{S}$. et al. Oxidation resistance of graphene-coated $\mathrm{Cu}$ and $\mathrm{Cu} / \mathrm{Ni}$ alloy. ACS Nano 5, 1321-1327 (2011).

41. Weatherup, R. S. et al. Long-term passivation of strongly interacting metals with single-layer graphene. J. Am. Chem. Soc. 137, 14358-14366 (2015).

42. Kravets, V. G. et al. Graphene-protected copper and silver plasmonics. Sci. Rep. 4, 5517 (2014).

43. Prasai, D., Tuberquia, J. C., Harl, R. R., Jennings, G. K. \& Bolotin, K. I. Graphene: corrosion-inhibiting coating. ACS Nano 6, 1102-1108 (2012).

44. Su, Y. et al. Impermeable barrier films and protective coatings based on reduced graphene oxide. Nat. Commun. 5, 4843 (2014).

45. Kim, K. et al. High-temperature stability of suspended single-layer graphene. Phys. Stat. Solidi RRL 4, 302-304 (2010).

46. Balandin, A. A. et al. Superior thermal conductivity of single-layer graphene. Nano Lett. 8, 902-7 (2008).

47. De Fazio, D. et al. High-mobility, wet-transferred graphene grown by chemical vapor deposition. ACS Nano 13, 8926-8935 (2019).

48. Giambra, M. A. et al. Wafer-scale integration of graphene-based photonic devices. ACS Nano 15, 3171-3187 (2021).

49. Albrecht, T. R. et al. Bit-patterned magnetic recording: Theory, media fabrication, and recording performance. IEEE Trans. Magn. 51, 0800342 (2015). 
50. Goohpattader, P. S. et al. Probing the role of $\mathrm{C}^{+}$ion energy, thickness and graded structure on the functional and microstructural characteristics of ultrathin carbon films (<2 nm). Tribo. Int. 81, 73-88 (2015).

51. Dwivedi, N. et al. Enhanced characteristics of pulsed DC sputtered ultrathin $(<2 \mathrm{~nm})$ amorphous carbon overcoats on hard disk magnetic media. Diam. Relat. Mater. 51, 14-23 (2015).

52. Bonaccorso, F. et al. Mater. Today 15, 564 (2012).

53. Bonaccorso, F., Sun, Z., Hasan, T. \& Ferrari, A. C. Nat. Photonics 4, 611 (2010).

54. Ferrari, A. C. \& Basko, D. M. Raman spectroscopy as a versatile tool for studying the properties of graphene. Nat. Nanotechnol. 8, 235-46 (2013).

55. Hussain, S., Bhatia, C. S., Yang, H. Y. \& Danner, A. Characterization of Capertures in a successful demonstration of heat-assisted magnetic recording. Opt. Lett. 40, 3444-3447 (2015).

56. Lagatsy, A. A. et al. $2 \mu \mathrm{m}$ solid-state laser mode-locked by single-layer graphene. Appl. Phys Lett. 102, 013113-1 (2013).

57. Cançado, L. G. et al. Quantifying defects in graphene via Raman spectroscopy at different excitation energies. Nano Lett. 11, 3190-6 (2011).

58. Ferrari, A. C. \& Robertson, J. Interpretation of Raman spectra of disordered and amorphous carbon. Phys. Rev. B 61, 14095 (2000).

59. Bruna, M. et al. Doping dependence of the raman spectrum of defected graphene. ACS Nano 8, 7432-7441 (2014).

60. Ferrari, A. C. et al. Raman spectrum of graphene and graphene layers. Phys. Rev. Lett. 97, 187401 (2006).

61. Mohiuddin, T. M. G. et al. Uniaxial strain in graphene by Raman spectroscopy: $G$ peak splitting, Gruneisen parameters and sample orientation. Phys. Rev. B 79, 205433 (2009).

62. Yoon, D., Son, Y.-W. \& Cheong, H. Strain-dependent splitting of the doubleresonance Raman scattering band in graphene. Phys. Rev. Lett. 106, 155502 (2011).

63. Rose, F. et al. Complete characterization by Raman spectroscopy of the structural properties of thin hydrogenated diamond-like carbon films exposed to rapid thermal annealing. J. Appl. Phys. 116, 123516 (2014).

64. Yang, H. et al. Significant Dzyaloshinskii-Moriya interaction at grapheneferromagnet interfaces due to the Rashba effect. Nat. Mater. 17, 605 (2018).

65. Ono, T. et al. Addition of Ru to $\mathrm{L}_{0}-\mathrm{FePt}$ thin film to lower Curie temperature. Appl. Phys. Express 9, 123002 (2016).

66. Calizo, I., Balandin, A. A., Bao, W., Miao, F. \& Lau, C. N. Temperature dependence of the Raman spectra of graphene and graphene multilayers. Nano Lett. 7, 2645-2649 (2007).

67. Erdemir, A. et al. Carbon-based tribofilms from lubricating oils. Nature 536, 67-71 (2016)

68. Prutton, M. \& Gomati, M. M. El. Scanning Auger Electron Microscopy (2006).

69. White, W. B. \& Minser, D. G. Raman spectra and structure of natural glasses. J. Non-Crystal. Solids 67, 45-59 (1984).

70. Gallant, D., Pezolet, M. \& Simard, S. Optical and physical properties of cobalt oxide films electrogenerated in bicarbonate aqueous media. J. Phys. Chem. B 110, 6871-6880 (2006).

71. Hadjiev, V. G., Iliev, M. N. \& Vergilov, I. V. The Raman spectra of $\mathrm{Co}_{3} \mathrm{O}_{4}$. J. Phys. C 21, L199-L201 (1988).

72. Banerjee, I., Kim, H. K. D., Pisani, D., Mohanchandra, K. P. \& Carman, G. P. Magnetic anisotropy and magnetodielectric coefficients in $\mathrm{Cr}_{2} \mathrm{O}_{3}$ and $\mathrm{Fe}_{0.4}$ $\mathrm{Cr}_{1.6} \mathrm{O}_{3}$. J. Alloys Compd 614, 305 (2014)

73. Rafiee, J. et al. Wetting transparency of graphene. Nat. Mater. 11, 217 (2012).

74. Kondo, H. Tribochemistry of Ionic Liquid Lubricant on Magnetic Media. Adv. Tribol. 2012, 526726 (2012).

75. Tomcik, B., Osipowicz, T. \& Lee, J. Y. Diamond-like film as a corrosion protective layer on the hard disk. Thin Solid Films 360, 173-180 (2000).

76. Bowden, F. P. \& Tabor, D. Mechanism of metallic friction. Nature 150, 197-199 (1942).

77. Krim, J. Resource Letter: FMMLS-1: Friction at macroscopic and microscopic length scales. Am. J. Phys. 70, 890 (2002).

78. Liu, X. et al. Mechanical properties of soft, electrodeposited Fe-Co-Ni films for magnetic recording heads. IEEE Trans. Magn. 38, 2231 (2002).

79. Riedl, A. et al. Tribological properties of $\mathrm{Al}_{2} \mathrm{O}_{3}$ hard coatings modified by mechanical blasting and polishing post-treatment. Wear 289, 9-16 (2012).

80. Rana, A. et al. Correlation of nanoscale behaviour of forces and macroscale surface wettability. Nanoscale 8, 15597-15603 (2016).

81. Li, S. et al. Nature 59, 541-545 (2016).

82. Li, Q., Lee, C., Carpick, R. W. \& Hone, J. The evolving quality of frictional contact with graphene. Phys. Status Solidi B 247, 2909-2914 (2010).
83. Tan, P. H. et al. The shear mode of multilayer graphene. Nat. Mater. 11, 294-300 (2012)

84. Lee, C., Wei, X. D., Kysar, J. W. \& Hone, J. Measurement of the elastic properties and intrinsic strength of monolayer graphene. Science 321, 385 (2008).

85. Berman, D., Erdemir, A. \& Sumant, A. V. Few layer graphene to reduce wear and friction on sliding steel surfaces. Carbon 54, 454-459 (2013).

86. Marchetto, D., Feser, T. \& Dienwiebel, M. Microscale study of frictional properties of graphene in ultra high vacuum. Friction 3, 161-169 (2015).

87. Zhuang, C. \& Liu, L. Atomic-scale friction modulated by potential corrugation in multi-layered graphene materials. J. Appl. Phys. 117, 114302 (2015).

\section{Acknowledgements}

We acknowledge funding from the National Research Foundation, Prime Minister's Office, Singapore under its Competitive Research Program (CRP Award No. NRF-CRP 4-2008-06), the EU Graphene Flagship, EU grant CareRAMM, ERC Grant Hetero2D, EPSRC Grants EP/K01711X/1, EP/ K017144/1, EP/N010345/1, EP/L016057/1, EU grant Neurofibres, the National Energy Research Scientific Computing Center, a DOE Office of Science User Facility, supported by the Office of Science of the U.S. Department of Energy under Contract No. DE-AC02-05CH11231, the Center for Nanoscale Materials by the U.S. Department of Energy, Office of Science, Office of Basic Energy Sciences, under Contract DE-AC02-06CH11357, the Exeter Time-Resolved Magnetism Facility (EXTREMAG, EPSRC Grant EP/R008809/1)

\section{Author contributions}

A.C.F. and C.S.B. conceived the idea, designed the project and supervised the work. N.D. and T.D. prepared the media substrates. N.D. applied lubricant. A.K.O., C.D., U.S., D.D.F., O.B., S.S., J.Z., and A.K.K. prepared different sets of graphene containing samples. N.D. performed tribological measurements. A.K.O. performed Raman spectroscopy and AFM. A.K.O. and G.S. performed the irradiation measurements. A.K.O. and P.S.K. performed MOKE measurements. R.J.Y. performed corrosion measurements. K.S., B.N., and S.K.R.S.S. performed MD simulations. N.D., A.K.O., R.J.Y., K.S., and B.N. analyzed the results with inputs from A.K.S., S.K.R.S.S., A.C.F. N.D., A.K.O. C.S.B., and A.C.F. wrote the manuscript. All the authors critically reviewed the manuscript. N.D. and A.K.O. contributed equally to this work.

\section{Competing interests}

The authors declare no competing interests

\section{Additional information}

Supplementary information The online version contains supplementary material available at https://doi.org/10.1038/s41467-021-22687-y.

Correspondence and requests for materials should be addressed to A.C.F.

Peer review information Nature Communications thanks Vinod Sangwan and the other anonymous reviewer(s) for their contribution to the peer review of this work.

Reprints and permission information is available at http://www.nature.com/reprints

Publisher's note Springer Nature remains neutral with regard to jurisdictional claims in published maps and institutional affiliations.

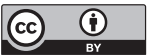

Open Access This article is licensed under a Creative Commons Attribution 4.0 International License, which permits use, sharing, adaptation, distribution and reproduction in any medium or format, as long as you give appropriate credit to the original author(s) and the source, provide a link to the Creative Commons license, and indicate if changes were made. The images or other third party material in this article are included in the article's Creative Commons license, unless indicated otherwise in a credit line to the material. If material is not included in the article's Creative Commons license and your intended use is not permitted by statutory regulation or exceeds the permitted use, you will need to obtain permission directly from the copyright holder. To view a copy of this license, visit http://creativecommons.org/ licenses/by/4.0/.

(C) The Author(s) 2021, corrected publication 2021 